\title{
The Hallmarks of Mathematical Oncology
}

\author{
Joshua Adam Bull and Helen Mary Byrne
}

\begin{abstract}
Over the past twenty-five years there has been an unparalleled increase in understanding of cancer biology. This transformation is exemplified by Hanahan and Weinberg's decision in 2011 to expand their original Hallmarks of Cancer from six traits to ten! At the same time, mathematical modelling has emerged as a natural tool for unravelling the complex processes that contribute to the initiation and progression of tumours, for testing hypotheses about experimental and clinical observations, and assisting with the development of new approaches for improving its treatment. This article starts by reviewing some of the earliest models of tumour growth and tumour responses to radiotherapy. Following Hanahan and Weinberg's lead, attention then focuses on how closer collaboration with cancer scientists and access to experimental data is stimulating the development of new and increasingly detailed models which account, for example, for tumour-immune interactions and immunotherapy. The article concludes by discussing the ways in which mathematical modelling is being integrated with experimental and clinical work and outlining how this could improve disease diagnosis and the delivery of effective personalised treatments to cancer patients. As such, the article serves as an introduction to mathematical modelling of cancer and its treatments, suitable for researchers seeking to enter the field.
\end{abstract}

\section{INTRODUCTION}

\section{A. The Hallmarks of Cancer}

C ANCER is a generic term for a group of diseases that can affect any part of the body. While tremendous progress has been made in increasing our understanding of how tumours grow and improving their response to treatment, the worldwide burden of cancer incidence and mortality continues to grow, with approximately 19.3 million new cases and 10.0 million cancer-related deaths in 2020 [1]. These statistics highlight the complex and diverse nature of cancer and the continued need to increase understanding of how cancers develop, and how treatments should be delivered to maximise the therapeutic benefit for individual patients.

Defining features of cancer include the rapid accumulation of abnormal cells that invade adjoining parts of the body and spread, or metastasise, to other organs. In 2000, Hanahan and Weinberg introduced a conceptual framework for understanding the diversity of cancer in terms of six defining features or hallmarks: sustaining proliferative signalling, evading growth suppressors, activating invasion and metastasis, enabling replicative immortality, inducing angiogenesis (i.e., the growth of new blood vessels from existing ones), and resisting cell death [2]. Hanahan and Weinberg proposed that transformation of normal cells to cancer cells requires the successive accumulation of multiple hallmarks, and that the cancer's diversity reflects the multiple ways in which they

J.A. Bull and H.M. Byrne are with the Mathematical Institute, University of Oxford, Oxford OX2 6GG, UK. E-mail: joshua.bull@maths.ox.ac.uk and helen.byrne@maths.ox.ac.uk.

Manuscript received August 01, 2021; revised August 01, 2021.

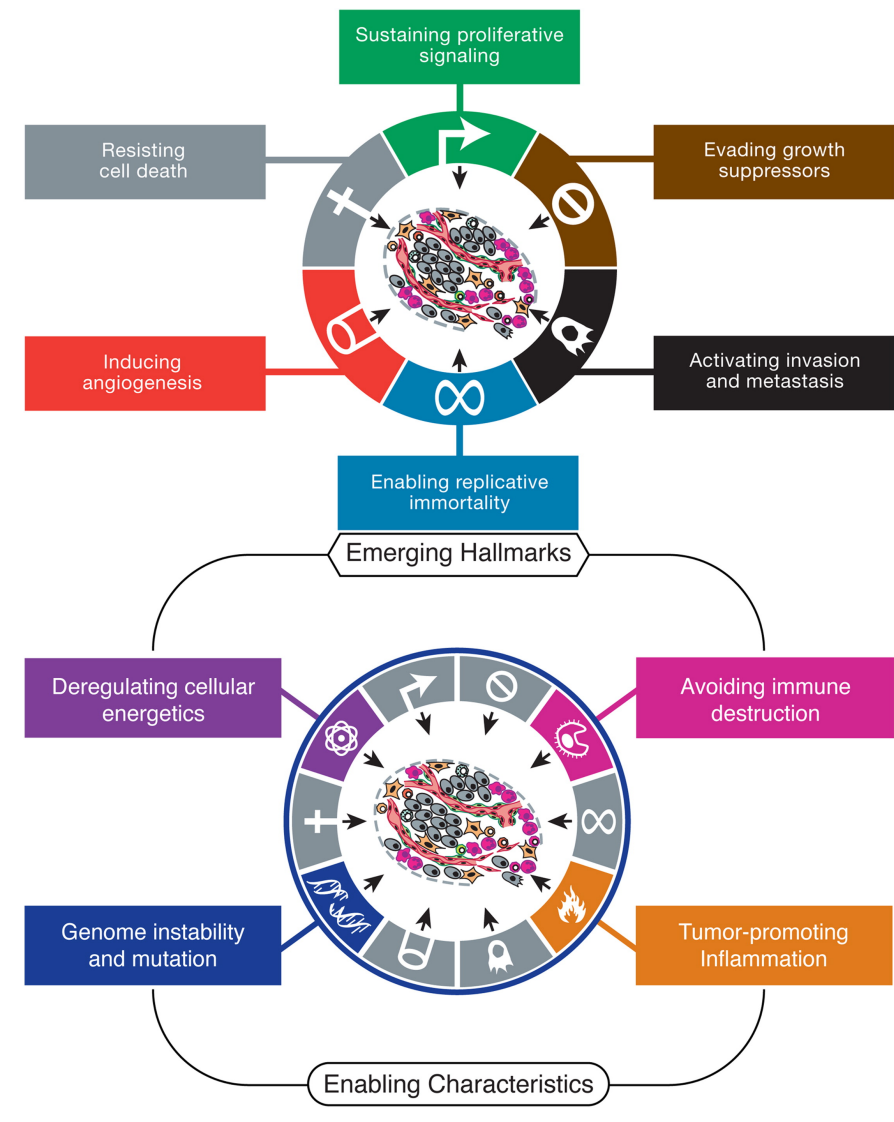

Fig. 1. The Hallmarks of Cancer, as described by Hanahan and Weinberg in [3]. Figure reproduced from [3], Figures 1 and 3.

may be acquired. In 2011, increased understanding of cancer biology led Hanahan and Weinberg to introduce four new hallmarks: avoiding immune destruction, deregulating cellular energetics, tumour-promoting inflammation, and genomic instability and mutation (see Figure 1 and [3]).

\section{B. The Hallmarks of Mathematical Oncology}

The Hallmarks of Cancer provide a conceptual framework for understanding different facets of cancer biology and why it is such a diverse disease. However, with verbal reasoning alone, it is difficult to understand how, for a particular individual, the order in which the hallmarks occur impacts their tumour's growth dynamics and/or response to treatment. The main aim of this article is to illustrate how mathematical modelling can provide a complementary framework for addressing these and related questions. Figure 2 shows the Hallmarks of Mathematical Oncology, a conceptual framework we introduce to characterise mathematical models. Each hallmark represents a modelling decision which determines the level of biological 


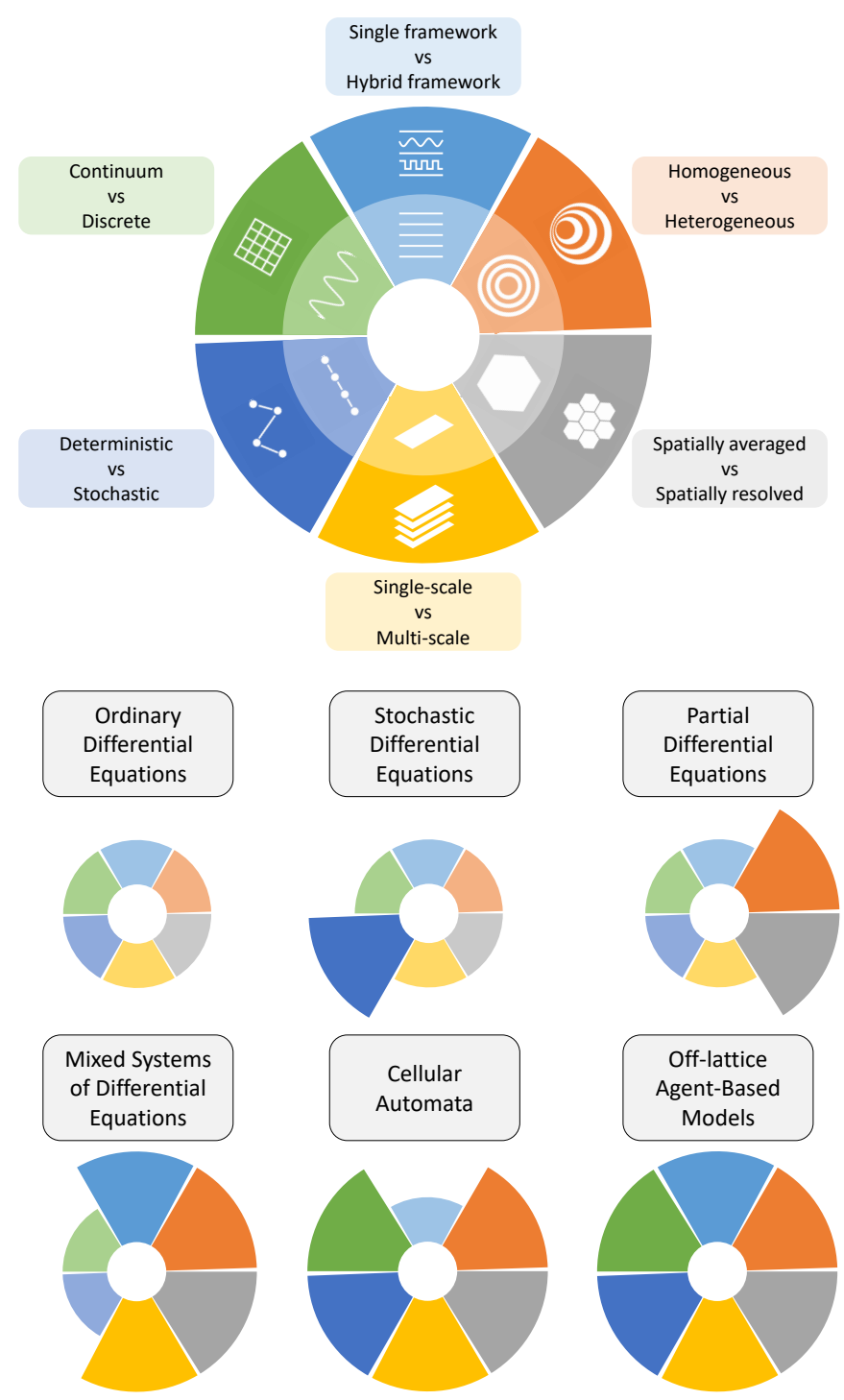

Fig. 2. Top: The Hallmarks of Mathematical Oncology: a schematic diagram illustrating the approaches used to develop mathematical models. Bottom: Series of glyphs showing how different modelling frameworks may incorporate different mathematical hallmarks. Approaches which require additional complexity in mathematical hallmarks may resolve more detailed biological processes than simpler frameworks.

detail considered, the complexity of generating model solutions, and the types of data that the model can reproduce.

We identify six key mathematical hallmarks, or decisions: single vs hybrid frameworks, homogeneity vs heterogeneity, spatially-averaged vs spatially-resolved, single-scale vs multiscale, deterministic vs stochastic, and continuum vs discrete. Different mathematical models of cancer combine these hallmarks in different ways, leading to models which may include more biological complexity but are more challenging to analyse and/or parameterise. The glyphs in the bottom panel of Figure 2 use the mathematical hallmarks to illustrate the increasing complexity of different modelling approaches. In this paper, we explain how these frameworks can be used to model the same biological phenomenon (responses to treatment with cancer radiotherapy) in increasing levels of detail.

By studying a series of increasingly detailed models, we aim also to demonstrate the additional insight that increased biological complexity and realism enable, and the need to balance these benefits against the costs. For example, with limited experimental data, it may not be possible to validate a complex model or to estimate values of its parameters.

The literature on mathematical oncology is now so large that, in limited space, we must be selective. We have chosen to focus on three modelling approaches that have been used to investigate solid tumour growth and tumour responses to radiotherapy (the following, excellent texts provide further information about these and alternative modelling approaches: $[4]-[6])$. At the same time, we explain how more detailed modelling frameworks allow more detailed consideration of the factors limiting the efficacy of radiotherapy to be investigated, and assessment of strategies for their compensation.

The remainder of this article is organised as follows. In Section II, we provide a brief introduction to cancer biology and radiotherapy, and outline the process by which mathematical models are developed, tested and refined. In Section III, we showcase simple models of solid tumour growth, formulated using ordinary differential equations, and explain how they can be adapted to predict radiotherapy responses. In Section IV, we introduce spatially-resolved models, formulated using partial differential equations, that can be used to investigate how spatial variation in cell density and nutrient levels impact the growth and composition of solid tumours. In Section V, we focus on agent-based models that can be used to investigate how the behaviour of individual cells influences the dynamics at the tissue or macroscale. For each modelling approach, we also explain how the models can be extended to include more biological realism and, in particular, to account for specific Hallmarks of Cancer. In Section VI, we outline the ways in which the different models are being integrated with experimental and clinical data. The paper ends in Section VII with some general conclusions and future perspectives.

\section{BiOlogicAl AND MATHEMATICAL BACKGROUND}

\section{A. Biological Background}

The multistage process by which normal cells transform into cancer cells, as embodied by the Hallmarks of Cancer, is associated with the progressive loss of function of a range of regulatory genes [2], [3]. These include repair genes that correct for mutations and DNA damage before cell division and tumour suppressor genes that signal for cell-cycle arrest or programmed cell death (apoptosis) if substantial genetic damage is detected. Uncontrolled cell division leads to the growth of (small) avascular tumours, which must recruit a new blood supply, via angiogenesis, to sustain their growth.

During avascular growth, nutrients are consumed by live, proliferating cells as they diffuse from the tumour periphery to its centre. Therefore, as an avascular tumour grows, nutrient levels at its centre decline until it reaches a critical size at which there is insufficient nutrient to sustain viable cells, and a central core of dead (necrotic) cellular material forms, its size increasing as the tumour continues to grow. Thus, 
a well-developed, avascular tumour comprises an outer rim of nutrient-rich, proliferating cells, a central core of nutrientstarved, necrotic debris, and an intermediate layer of oxygenpoor (or hypoxic) cells which are quiescent (i.e., viable but non-proliferating). Since diffusion controls nutrient delivery to, and the removal of waste products from, avascular tumours [7], the diameter to which they may grow is limited to several millimetres and they are relatively harmless. By contrast, vascular tumours are life-threatening for two main reasons. First, being connected to the host's blood supply, they have access to an almost limitless supply of nutrients. Their consequent rapid growth may impair the function of neighbouring vital organs. Secondly, tumour fragments that break away from the primary tumour and enter blood vessels may be transported to other parts of the body where they establish secondary tumours (metastases) that further jeopardise the host. The switch from avascular to vascular growth is achieved by activating angiogenesis, a hallmark of cancer [8].

During angiogenesis, the tumour cells secrete multiple diffusible factors (e.g. vascular endothelial growth factor and tumour necrosis factor- $\beta$ ), known collectively as angiogenic factors, that stimulate endothelial cells from nearby blood vessels to proliferate and migrate towards the tumour, forming new vessels that supply it with the nutrients required for continued growth. Invasion of healthy tissue is another hallmark of cancer: contact with the healthy tissue stimulates the tumour cells to produce matrix-degrading enzymes (e.g., matrix metallo-proteases) that create spaces into which the tumour cells can migrate [9]. At a cellular level, the sequence of events needed to establish a well-developed, vascularised tumour may be associated with mutations in a variety of genes. For example, it has been estimated that up to $50 \%$ of tumours harbour mutations in the tumour suppressor gene p53, and these mutations have been linked with angiogenesis and cell immortality, two Hallmarks of Cancer [10].

The above discussion underscores the complex and multiscale nature of solid tumour growth, with multiple biophysical processes interacting across a wide range of spatial and temporal scales. At the subcellular scale individual cells produce proteins which regulate their behaviour, at the cellular scale normal and cancerous cells compete for space and nutrients, while behaviours at the macroscale include vascular remodelling, blood flow and nutrient transport. Now, in response to environmental stresses such as hypoxia, tumour cells may alter their subcellular behaviour by slowing (or halting) the rate at which they progress through the cell cycle and/or altering the rate at which they produce angiogenic factors such as VEGF. When released by the cell, the angiogenic factors diffuse through the tissue until they reach neighbouring vessels where they stimulate the formation of new blood vessels and, thereby, increase the supply of nutrients to the tumour. In this way, phenomena at the subcellular and macroscale may interact. We will explore these ideas further in section $\mathrm{V}$, where we introduce multiscale models of tumour growth.

\section{B. Radiotherapy}

During radiotherapy, beams of ionising particles are directed at cancer cells to kill them or slow their growth by inflicting single- and double-strand breaks on their nuclear DNA. A 1 Gy $(1 \mathrm{~Gy}=1$ Gray $=1$ Joule $/ \mathrm{kg})$ dose of gamma radiation causes approximately 1,000 single-strand breaks, and 20-25 double-strand breaks [11]. Cell survival following radiation depends on fully functioning DNA damage repair mechanisms, with cell death triggered only when double-strand breaks are not properly repaired. Typically, only $1-2 \%$ of double-strand breaks are lethal and the number of cells that survive exposure to radiotherapy is highly dependent on the dose rate [12]. Radiotherapy is most effective when it elicits favourable, differential responses in the host and cancer cells, such as when the host cells have lower proliferation rates and better repair mechanisms than tumor cells.

Radiotherapy may be administered prior to surgery to shrink a tumour, during surgery to minimise damage to surrounding healthy tissue [13], and post-surgery to prevent regrowth of any residual tumour cells. It is often combined with chemotherapy [14] and, increasingly, with treatments such as hyperthermia [15], virotherapy [16] and immunotherapy [17]. Typical radiotherapy protocols last for 6-8 weeks, with treatment administered 5 days per week, with rest periods at weekends.

When directing radiotherapy at cancer cells, it is important to limit the damage to adjacent healthy tissue. Typically, treatment aims to maximise the cancer cell kill while ensuring the damage sustained by neighbouring healthy cells is within tolerable levels. In practice, additional factors also limit the efficacy of radiotherapy. For example, tumours may fail to respond to radiotherapy due to intrinsic or acquired (i.e., treatmentinduced) resistance, with cancer stem cells viewed as one source of such resistance. Hypoxia, arising from the abnormal and poorly functioning tumour vasculature, is also a barrier to the efficacy of radiotherapy, with hypoxic cells estimated to be up to 3 times more radioresistant than well-oxygenated cells. Novel strategies being designed to boost tumour oxygen levels and, thereby, to enhance tumour radiosensitivity, include hyperbaric oxygen and metabolic inhibitors [18], [19].

The linear-quadratic (LQ) model is widely used to calculate the fraction $S F$ of cells that survive exposure to $n$ fractions of radiotherapy with dose $d$, delivered over the time period $0<t<t_{\text {end }}$

$$
S F=\frac{V\left(t=t_{\text {end }}\right)}{V(t=0)} \equiv \exp \left(-n\left(\alpha d+\beta d^{2}\right)\right),
$$

where the positive constants $\alpha$ and $\beta$ are tumour-specific radiosensitivity parameters and $V(t=0)$ and $V\left(t=t_{\text {end }}\right)$ denote the initial and final tumour volumes [20].

When the survival fraction is plotted on a log scale, we observe a quadratic dose response. The response is dominated by the linear $(\alpha)$ term at low doses, and by the quadratic $(\beta)$ term at higher doses, with the ratio $\alpha / \beta$ representing the dose at which the linear and quadratic terms are equal. Thus, the cell kill for cells with high $\alpha / \beta$ ratios remains relatively constant with increasing dose, and increases markedly for those with low $\alpha / \beta$ ratios. We may interpret the linear term as describing cell death from a 'single hit' (i.e., lethal damage caused by a single radiation track) while the quadratic term describes cell death due to 'multiple hits.' In practice, the cell death 
induced by exposure to radiotherapy occurs over a finite time period: the LQ model accounts for these dynamics implicitly, quantifying the total cell kill in terms of the radiotherapy dose $d$ and the values of the parameters $\alpha$ and $\beta$, and not explicitly resolving the temporal dynamics.

While some authors have derived the LQ model from a mechanistic perspective (e.g., relating the dose to the numbers of single and double stranded DNA breaks, and then relating the numbers of such DNA breaks to the cell kill) [21], it was originally developed to provide a phenomenological description of experimental observations of the dose-response relationship. Although the LQ model is widely used to predict tumour responses to radiotherapy, it has several shortcomings. For example, in its simplest form, the LQ model does not account explicitly for the five R's of radiotherapy: $\underline{\text { Repair, }} \underline{\mathrm{R}} \mathrm{e}-$ population, $\underline{\mathrm{R}} \mathrm{e}$-distribution of cells around the cell cycle, $\underline{\mathrm{R}} \mathrm{e}-$ oxygenation and radioResistance [22]. While the LQ model has been extended to account for these effects [23], [24], the relationships between dose and the 5 R's are complex and are often neglected - indeed, some argue that results generated from more complex dose-response models yield results that are equivalent to those of the original LQ model [21]. A further weakness of the LQ model is that it lacks explicit timedependence, focussing only on the total cell kill achieved by a dose $d$ of radiotherapy. Alternative models have been proposed which explicitly account for time-dependent responses to treatment (e.g., [25]-[27]).

The mathematical models introduced in this paper will illustrate how the 5 R's of radiotherapy can be modelled, through appropriate choices of the Hallmarks of Mathematical Oncology.

\section{Modelling Approaches}

Statistical techniques, applied to experimental data, can reveal correlations between observable phenomena. Establishing why such correlations arise requires the statement of hypotheses that propose which physical processes are involved and how they interact. The biological experiments needed to verify these hypotheses can be time-consuming and challenging to undertake. In such circumstances, mathematical models can be used to test the consistency of the hypotheses. In particular, if a mathematical model is unable to reproduce the observed phenomena, then the hypotheses should be revised before continuing. Mathematical models can also improve experimental design by highlighting which data should be collected to test a particular theory.

Figure 3 serves as a guide to the stages involved in developing and refining mathematical models. It also shows that mathematical modelling is an iterative process, whose success relies on continued multidisciplinary collaboration between experimentalists and theoreticians.

While Figure 3 provides a roadmap for developing, testing and exploiting mathematical models, it sidesteps a key aspect of model building: deciding what mathematical approach or framework should be used to formulate the mathematical model. Many alternative approaches can be used, as illustrated by the mathematical hallmarks outlined in Figure 2 , the models

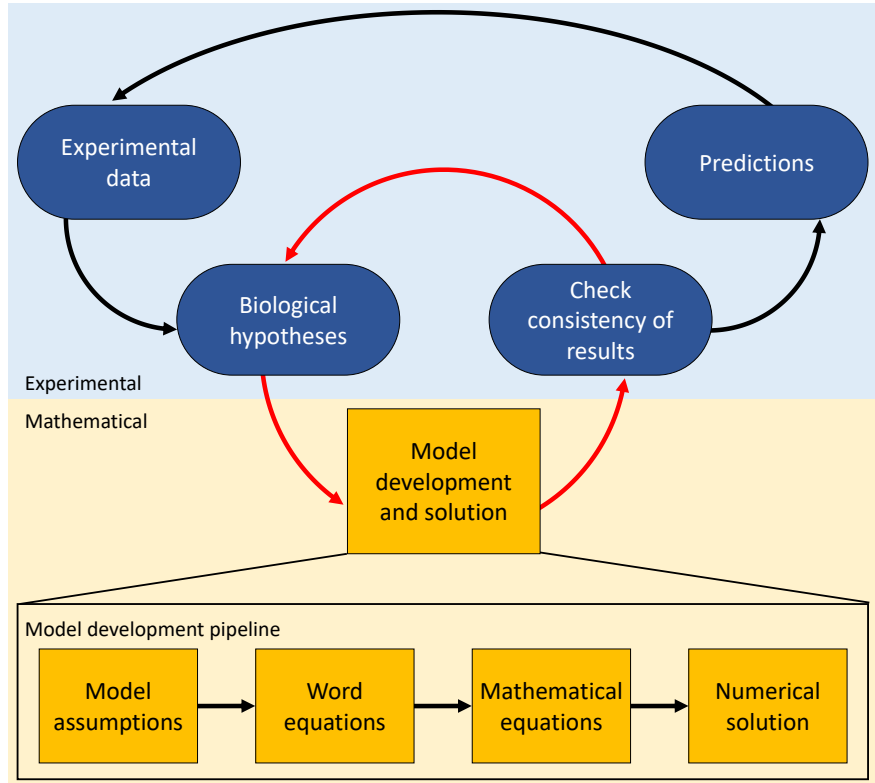

Fig. 3. A schematic diagram highlighting the iterative and multidisciplinary nature of mathematical modelling. Experimental data guide the generation of biological hypotheses that can be formalised in mathematical models. Model validation involves establishing consistency between model solutions and the experimental data. If this is not obtained, then the hypotheses and models must be revised until qualitative and quantitative agreement is reached. Parameter values can be estimated by fitting the model to the data and the model then used to generate experimentally testable predictions.

may be deterministic, comprising systems of time-dependent ordinary and/or partial differential equations, or they may be stochastic, accounting for random effects; they may be based on physical principles (e.g., Newton's laws of mechanics) or phenomenological in nature; they may focus on processes that happen on a specific spatial and/or temporal scale (e.g., subcellular signalling pathways) or they may be multiscale, coupling processes acting at the subcellular, cellular and tissue scales. With such an array of choices, it can be difficult to know how to proceed. In practice, decision-making should be guided by the questions that the modelling is designed to address and the quality and quantity of data available for model testing and validation.

In the remainder of this article, we aim to clarify these concepts by introducing a series of modelling approaches, outlining the assumptions on which they are based, highlighting the insight that they can provide, their possible extensions and their limitations. We start by focussing on arguably the simplest class of mathematical models: those that can be formulated using ordinary differential equations.

\section{ORdinARY DifFEREnTIAL EQUation MODELS}

In this section, we introduce several idealised models of solid tumour growth. Spatial effects are neglected and the models formulated as systems of time-dependent ordinary differential equations (ODEs). This corresponds to the simplest selection of mathematical hallmarks in Figure 2, with the tumour viewed as a spatially uniform and homogeneous cell population. We explain how models of this type may be used 
to determine how a tumour's volume changes over time, and integrated with models of cell kill due to radiotherapy in order to predict its impact and to compare different protocols for its delivery.

\section{A. Tumour Growth Models}

Arguably the simplest model that describes how the volume of a solid tumour $V$ evolves over time is the exponential growth law which states

$$
\frac{d V}{d t}=\lambda V, \quad \text { with } V(t=0)=V_{0},
$$

with solution

$$
V(t)=V_{0} e^{\lambda t} .
$$

In Equation (2), $\lambda>0$ and $V_{0}$ represent the net growth rate of the tumour and its initial volume, respectively. In this model, there are no growth constraints: all nutrients and growth factors are assumed to be in abundance. As a result, the model predicts unbounded growth of the tumour.

Whilst providing an accurate description of the early stages of a tumour's development, the exponential growth law fails to describe the reduced growth rates and eventual growth saturation observed when avascular tumours are cultured in vitro or when vascular tumours develop in vivo. In practice, the tumour's growth rate slows because, as it increases in size, competition for nutrients and other vital resources, such as space, can no longer be neglected. A simple modification of (2) which accounts for competition for resources (without specifying what those resources are) is the logistic growth law:

$$
\frac{d V}{d t}=\lambda V\left(1-\frac{V}{K}\right), \quad \text { with } V(t=0)=V_{0}>0,
$$

with solution

$$
V(t)=\frac{K V_{0}}{V_{0}+\left(K-V_{0}\right) e^{-\lambda t}}
$$

where $K>0$ represents the population's carrying capacity. Thus, at large times, the tumour volume $V(t)$ approaches the carrying capacity, $K$.

Whilst equation (3) predicts exponential growth of small tumours and growth saturation when the tumour reaches its carrying capacity $(V=K)$, we note that it has only two parameters, $\lambda$ and $K$. These parameters determine the tumour volume at which growth saturates $(K)$ and its growth rate. In practice, when fitting to experimental data, a model which offers greater flexibility in the growth dynamics may be desirable. For example, the generalised logistic model extends the logistic model by introducing an additional parameter, $\theta>0$, which allows greater flexibility over the growth dynamics. This model is given by

$$
\frac{d V}{d t}=\frac{\lambda}{\theta} V\left[1-\left(\frac{V}{K}\right)^{\theta}\right], \quad \text { with } V(t=0)=V_{0},
$$

with solution

$$
V(t)=K\left(\frac{V_{0}^{\theta}}{V_{0}^{\theta}+\left(K^{\theta}-V_{0}^{\theta}\right) e^{-\lambda t}}\right)^{1 / \theta} .
$$

A

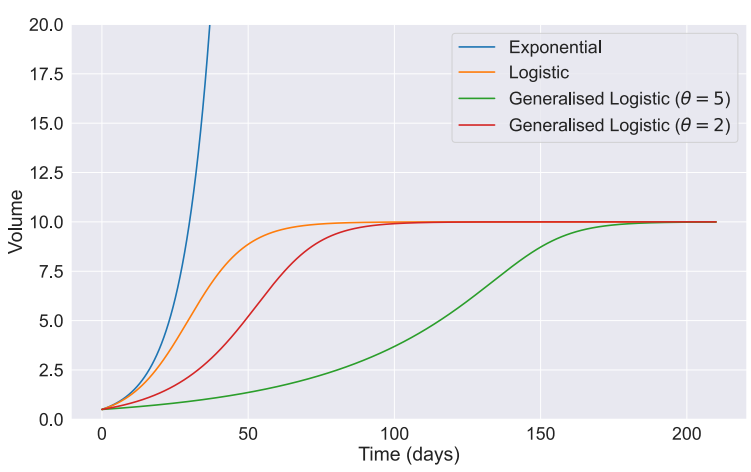

B

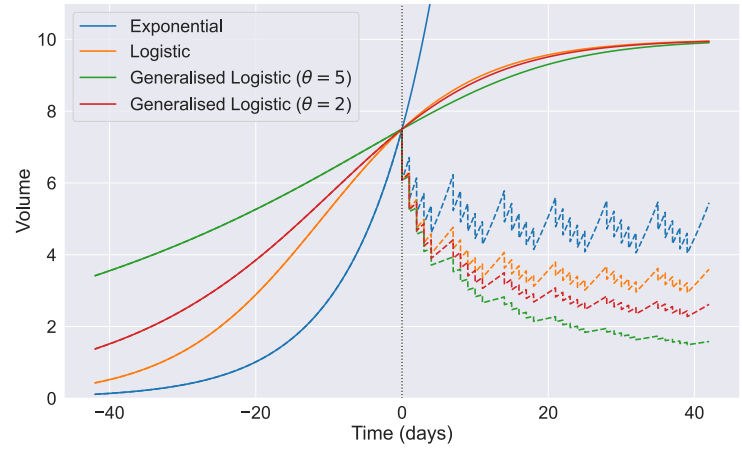

Fig. 4. Series of curves showing how the choice of growth law influences a tumour's growth dynamics (Panel A) and response to radiotherapy (Panel B). Key: exponential growth law (blue, $\lambda=0.1$ ); logistic growth law (orange, $\lambda=0.1, K=10$ ); generalised logistic growth law (green, $\lambda=0.1, K=$ $10, \theta=5$; red, $\lambda=0.1, K=10, \theta=2$ ). Panel B: Solid lines show untreated tumour growth, and dashed lines show the effect of treating the tumour with daily doses of 2 Gy of radiotherapy on weekdays beginning when each model reaches $v=7.5$ at $t=0$ (cell kill based on the linear quadratic model, with $\alpha=0.01, \beta=0.001)$.

We remark that equation (4) reduces to (3) when $\theta=1$.

In Figure $4 \mathrm{~A}$ we compare the three models described above, presenting plots of the growth dyanmics defined by equations (2), (3) and (4) for fixed values of $\lambda, K$, two values of $\theta$ and the same value of $V_{0}$, the initial tumour volume. Many other growth laws have been used to describe solid tumour growth [28]. Increasing the number of model parameters results in more flexible growth dynamics, and therefore a better fit to clinical or experimental data. However, using more complex models can result in overfitting and hence reduce the biological insight available from a model. To counter this, model selection methods such as Akaike's Information Criteria (AIC) and Bayesian Information Criteria (BIC) have been developed in order to balance the fit of a model against model complexity (see, e.g., [29]).

A common weakness of the ODE models introduced in this section is the difficulty of relating the system parameters (e.g., $\lambda, K$ and $\theta$ in equation (4)) to the behaviour of individual cells. We return to this matter in sections IV and V where we discuss spatially-structured models of tumour growth. First, we explain how the ODE models can be used to gain preliminary insight into how tumours respond to radiotherapy. 


\section{B. Tumour Growth and Radiotherapy}

For simplicity, we will consider a tumour which undergoes exponential growth in the absence of treatment (see Equation (2). We suppose that $n$ fractions of radiotherapy, with dose $d$, are administered at times $t_{i}(i=1,2,3, \ldots, n)$, that the resulting cell kill is instantaneous, and that the fraction $S F$ of cells that survive each dose of radiotherapy can be described by the LQ model (see Equation (1)). Under these assumptions, it is possible to show that the following ODE describes exponential growth between fractions and cell kill due to radiotherapy [30]:

$$
\frac{d V}{d t}=\lambda V-\sum_{i=1}^{n}\left(\alpha d+\beta d^{2}\right) V \delta\left(t-t_{i}\right),
$$

where $V(t=0)=V_{0}>0$ and $\delta(t)$ denotes the Dirac delta function (for an arbitrary smooth function, $f(t)$, the delta function is defined so that $\int_{-\infty}^{+\infty} f(t) \delta\left(t-t_{i}\right) d t=f\left(t_{i}\right)$ ). Solving Equation (5) subject to $V(t=0)=V_{0}>0$, we obtain

$$
\begin{aligned}
V(t) & =V\left(t_{i}^{+}\right) e^{\lambda\left(t-t_{i}\right)} \text { for } t_{i}^{+} \leq t \leq t_{i+1}^{-}, \\
\text {where } V\left(t_{i}^{+}\right) & =V\left(t_{i}^{-}\right) e^{-\left(\alpha d+\beta d^{2}\right)},
\end{aligned}
$$

and $V\left(t_{i}^{-}\right)$and $V\left(t_{i}^{+}\right)$denote the tumour volumes immediately before and after radiotherapy, and $V\left(t_{i}^{+}\right) / V\left(t_{i}^{-}\right) \equiv S F$ (see Equation (1)). Combining Equations (6) and (7) we deduce further that

$$
S F=\frac{V\left(t_{n}^{+}\right)}{V(0)} \equiv \underbrace{e^{\lambda t_{n}}}_{\begin{array}{c}
\text { contribution } \\
\text { due to growth }
\end{array}} \cdot \underbrace{e^{-n\left(\alpha d+\beta d^{2}\right)}}_{\begin{array}{c}
\text { contribution due } \\
\text { to radiotherapy }
\end{array}} .
$$

In particular, if growth is neglected (i.e., $\lambda=0$ ) then Equation (8) reduces to Equation (1). Thus, Equation (8) makes explicit how, by coupling the LQ model with an ODE model for (exponential) tumour growth it is possible to extend the LQ model to account for the combined effects of tumour growth and cell kill due to radiotherapy.

By suitable rearrangement of Equations (3) and (4), it is possible to combine cell kill due to radiotherapy with the logistic and generalised logistic growth laws in a similar manner. The corresponding ODEs are

$$
\frac{d V}{d t}=\lambda V\left[1-\left(\frac{V}{K}\right)^{\theta}\right]-\sum_{i=1}^{n}\left(\alpha d+\beta d^{2}\right) V \delta\left(t-t_{i}\right)
$$

with $V(t=0)=V_{0}>0$, and $\theta=1$ for the logistic growth law. The simulation results presented in Figure 4 $\mathrm{B}$ show how radiotherapy affects tumour growth, for the three models introduced in subsection III.A. Each model exhibits the same, "saw-tooth" dynamics, with tumour regrowth between radiotherapy doses and rapid (instantaneous) reductions in tumour volume when radiotherapy is administered. While the qualitative behaviour for each model is similar, for this set of parameter values, the long time dynamics differ: the exponential growth model predicts the smallest reduction in tumour volume and the two generalised logistic models the largest reduction. The variability in the model predictions highlights the importance of being able accurately to model a tumour's growth dynamics in order to accurately predict its response to radiotherapy.

The simulation results presented in Figure 4 assume that the cell survival fraction following radiotherapy is governed by the LQ model. While the LQ model is widely used in the clinic, we emphasise that it is phenomenological. Other authors have proposed alternative models of radiotherapy. Zahid, Mohsin, Mohamed, et al. [31] propose a novel approach in which radiotherapy doses instantaneously reduce the carrying capacity of the tumour in the logistic model, rather than directly affecting the tumour volume. In another example, Prokopiou et al. [32] proposed the following model for tumour response to radiotherapy:

$$
\frac{d V}{d t}=\lambda V\left(1-\frac{V}{K}\right)-\sum_{i=1}^{n} \gamma V\left(1-\frac{V}{K}\right) \delta\left(t-t_{i}\right),
$$

where $\gamma$ is a parameter describing the response to radiotherapy. Equation (10) assumes that the tumour undergoes logistic growth, and that the instantaneous cell kill due to radiotherapy is proportional to the logistic growth rate. A key assumption underpinning this model is that, when a tumour reaches its carrying capacity, the proportion of cells which are actively proliferating approaches zero. Since radiotherapy is more effective at killing proliferating cells, the cell kill also reduces as the tumour approaches its carrying capacity. These assumptions contrast with those for the LQ model, which assumes that the cell kill is proportional to the tumour volume when radiotherapy is administered (and does not reduce as the tumour approaches its carrying capacity). Comparison of Equations (5) and (10) illustrates the importance of understanding the assumptions on which a mathematical model is based. In particular, a tumour that approaches its carrying capacity because its (positive) rates of cell proliferation and cell death balance will remain sensitive to radiotherapy as it approaches its carrying capacity. By contrast, a tumour in which the proportion of proliferating cells diminishes as it approaches its carrying capacity will become less radiosensitive as it approaches its carrying capacity.

\section{Model extensions}

A common, simplifying assumption of the above ODE models is tumour homogeneity, both with regard to cell phenotypes and spatial variation across the tumour. In practice, however, tumours exhibit spatial and cellular heterogeneity. In Sections IV and V, we introduce models that account for spatial heterogeneity. First, we outline how ODE models can be adapted to account for tumour heterogeneity and to investigate the impact of several Hallmarks of Cancer (e.g., inducing angiogenesis, resisting cell death, tumour promoting inflammation and avoiding immune destruction: see Figure 1 on tumour growth and response to radiotherapy.

While it is possible to develop ODE models that account for vasculature, immune cells and different subpopulations of healthy and cancerous cells, mathematical models typically focus on a limited number of species. For example, in [33] the classical Lotka-Volterra model is used to study the dynamics of co-cultured populations of radiosensitive and radioresistant 
tumour cells and to investigate whether exposure to radiotherapy alters the way in which they interact. In the absence of radiotherapy, the governing equations can be written as

$$
\begin{aligned}
\frac{d V_{S}}{d t} & =\lambda_{S} V_{S}\left(1-\frac{V_{S}}{K_{S}}-\gamma_{R} \frac{V_{R}}{K_{S}}\right), \\
\frac{d V_{R}}{d t} & =\lambda_{R} V_{R}\left(1-\frac{V_{R}}{K_{R}}-\gamma_{S} \frac{V_{S}}{K_{R}}\right),
\end{aligned}
$$

where $V_{S}(t)$ and $V_{R}(t)$ represent the volumes of the control and resistant populations, $\lambda_{S}$ and $\lambda_{R}$ their growth rates, $K_{S}$ and $K_{R}$ their carrying capacities, so that if $V_{R}=0$ then the radiosensitive cells undergo logistic growth (and vice versa). The parameters $\gamma_{S}$ and $\gamma_{R}$ describe the effect that the radiosensitive cells have on the resistant cells (and vice versa): if $\gamma_{S}>0$ and $\gamma_{R}>0$ then the interactions are competitive, i.e., the presence of one cell type inhibits the growth of the other. If $\gamma_{S}<0$ and $\gamma_{R}<0$, then interactions are mutualistic, since the presence of one cell type enhances the growth of the other. Finally, if $\gamma_{S}$ and $\gamma_{R}$ have opposite signs then the interactions are antagonistic, since interactions in one direction are beneficial and in the other are detrimental (e.g., if $\gamma_{R}<0<\gamma_{S}$ then growth of sensitive cells is enhanced by resistant cells, while resistant cells are hindered by sensitive cells).

Similar ODE models have been proposed to describe the impact on tumour growth and radiotherapy responses of the vasculature [34], [35], and the immune system [36], [37], and also to explore the efficacy of treatments that combine radiotherapy with, for example, chemotherapy [38], anti-angiogenic therapy [35], and virotherapy [39].

The LQ model assumes that cell death following exposure to radiotherapy is instantaneous and that any dead material is eliminated from the tumour immediately. These assumptions give rise to the saw-tooth dynamics depicted in Figure 4. In practice, such dead material is removed (or phagocytosed) by immune cells over a finite time period, leading to behaviours which differ from the saw-tooth dynamics. This can be addressed by including multiple model compartments which account for viable cells and lethally damaged cells [40]. For example, Lewin et al. have developed an ODE model which distinguishes viable tumour volume $(V(t))$ and dead or necrotic material $(N(t))$ and accounts for degradation of dead material [41]:

$$
\begin{aligned}
& \frac{d V}{d t}=\lambda V\left(1-\frac{V}{K}\right)-\eta V-\gamma \sum_{i=1}^{n} V \delta\left(t-t_{i}\right), \\
& \frac{d N}{d t}=\eta V-\zeta N+\gamma \sum_{i=1}^{n} V \delta\left(t-t_{i}\right) .
\end{aligned}
$$

In addition to undergoing logistic growth, the viable tumour cells undergo necrosis at a constant rate $\eta$. The necrotic material undergoes exponential decay at constant rate $\zeta$. Fractionated radiotherapy, delivered at times $t_{i}$, causes instantaneous mass exchange between the viable and necrotic compartments. The dead material is unaffected by radiotherapy and, so, radiation-induced cell death is proportional to the viable tumour volume, $V$, with death rate $\gamma$, which contrasts with the corresponding terms using in Equations (9) and
(10) where the logistic term is assumed to encompass the population of cells sensitive to irradiation. Extensive model simulations reveal how their two compartment model may better capture the full range of dynamics observed in vivo (see Section VI and Figure 10 for more details).

When using ODE models to simulate tumour shrinkage during treatment with radiotherapy, we must be mindful of their limitations. In particular, as the tumour volume becomes very small (e.g., below the size of ten or fewer cells), stochastic effects become important and the validity of using differential equations to describe the system dynamics breaks down. In such cases, stochastic models can be developed to describe the system dynamics and to predict the probability of tumour elimination in response to treatment [25].

\section{PDE MODELS}

Many cell functions depend on their microenvironment. For example, in well-oxygenated regions, tumour cells proliferate rapidly and are highly sensitive to radiotherapy. By contrast, in hypoxic regions, cells reduce, or halt, their cell cycle and are less sensitive to radiotherapy. ODE models are unable to account for the effects that environmental and cellular heterogeneity have on a tumour's spatial composition, its growth dynamics and its response to treatment. In this section we introduce two models which use partial differential equations (PDEs) to investigate the effects that spatial variation in the local microenvironment and the tumour cell density have on tumour growth. We term these models the Greenspan model [42] and the proliferation-invasion model [43].

\section{A. The Greenspan model}

Greenspan's model is one of the earliest models to account for spatial heterogeneity. It considers a radially-symmetric tumour whose growth is regulated by a single, diffusible growth factor that is supplied externally (here taken to be oxygen). The distribution of the growth factor within the tumour regulates its local behaviour, with volumetric growth where cell proliferation exceeds cell death (and vice versa). By integrating these contributions over the tumour volume, we arrive at the following equation which relates the time evolution of the tumour volume $V(t)$ to the oxygen concentration $c(r, t)$ within the tumour volume:

$$
\frac{d V}{d t}=\int_{\partial V} F(c) d V
$$

where $F(c)$ describes the dependence on local oxygen levels of the net growth rate. Under the assumption of radial symmetry, $V(t)=\frac{4}{3} \pi R^{3}(t)$, where $R(t)$ denotes the position of the tumour boundary, and Equation 15 reduces to give

$$
\frac{d R}{d t}=\frac{1}{R^{2}} \int_{0}^{R(t)} F(c) r^{2} d r,
$$

with $R(0)=R_{0}$ prescribed. The oxygen distribution is governed by a reaction-diffusion equation of the form

$$
\frac{\partial c}{\partial t}=\frac{1}{r^{2}} \frac{\partial}{\partial r}\left(r^{2} \frac{\partial c}{\partial r}\right)-G(c),
$$


with boundary and initial conditions of the form

$$
\begin{array}{r}
c(R(t), t)=c_{\infty} \text { on } r=R(t), \\
\frac{\partial c}{\partial r}=0 \text { at } r=0, \\
c(r, 0)=c_{0}(r) \text { for } 0 \leq r \leq R_{0},
\end{array}
$$

where $G(c)$ and $c_{\infty}$ denote the oxygen consumption rate and the assumed constant oxygen concentration on the tumour boundary, and $c_{0}(r)$ is the oxygen distribution within the tumour at $t=0$.

For simplicity (and without appropriate data to justify more complex functions), Greenspan and many others have used idealised functional forms for $F(c)$ and $G(c)$ of the form

$$
\begin{aligned}
& F(c)=s_{0} c H\left(c-c_{H}\right)-\mu_{A}-\mu_{N} H\left(c_{N}-c\right), \\
& G(c)=\Gamma H\left(c-c_{N}\right),
\end{aligned}
$$

where $H$ is the Heaviside function $(H(x)=1$ if $x>0$, $H(x)=0$ otherwise) and $c_{H}$ and $c_{N}$ are oxygen thresholds below which cells become hypoxic $\left(c \leq c_{H}\right)$ and necrotic $\left(c \leq c_{N}<c_{H}\right)$. Thus, in well-oxygenated regions, where $c>c_{H}$, cells proliferate, at a rate proportional to their local oxygen concentration, with constant of proportionality $s_{0}$, undergo natural or apoptotic cell death at rate $\mu_{A}$, and consume oxygen at a constant rate $\Gamma$. In low oxygen regions (i.e., under hypoxia), where $c_{N}<c<c_{H}$, the cells do not proliferate or consume oxygen, but they undergo necrosis. In very low oxygen regions, where $c \leq c_{N}$, cells are unable to remain viable and they undergo apoptotic and necrotic cell death, the latter at rate $\mu_{N}$.

With cell proliferation and necrotic cell death occurring in distinct spatial regions, we can see how spatial heterogeneity can be incorporated within a PDE framework. Indeed, it is possible to identify the sizes of the proliferating, hypoxic and necrotic regions by tracking the positions $r=R_{H}(t)$ and $r=$ $R_{N}(t)$ at which the oxygen concentration attains the hypoxic and necrotic threshold values:

$$
\begin{aligned}
& R_{H}=0 \text { if } c>c_{H} \forall r \text { and otherwise } c\left(R_{H}, t\right)=c_{H}, \\
& R_{N}=0 \text { if } c>c_{N} \forall r \text { and otherwise } c\left(R_{N}, t\right)=c_{N} .
\end{aligned}
$$

Following [44], we can exploit the spatial heterogeneity of the Greenspan model, to develop a description of tumour response to radiotherapy, which accounts for differential radiosensitivity in well-oxygenated and hypoxic regions: we replace Equation (1) by a local survival fraction, $S F(c)$, where

$$
S F(c)=\exp \left(-\left(\alpha \cdot \frac{d}{\mathrm{OER}(c)}+\beta \cdot \frac{d^{2}}{\mathrm{OER}^{2}(c)}\right)\right),
$$

where the oxygen enhancement factor $\operatorname{OER}(c)$ is given by

$$
\operatorname{OER}(c)= \begin{cases}1, & \text { if } c>c_{H}, \\ 3 & \text { if } c_{N} \leq c \leq c_{H} .\end{cases}
$$

In Figure 5 we present typical model solutions, showing tumour responses to a 6-week fractionation protocol where 2 Gy fractions are delivered daily Monday-Friday. As for the ODE models, the tumour radius undergoes saw-tooth dynamics, although we can now see how the tumour's spatial
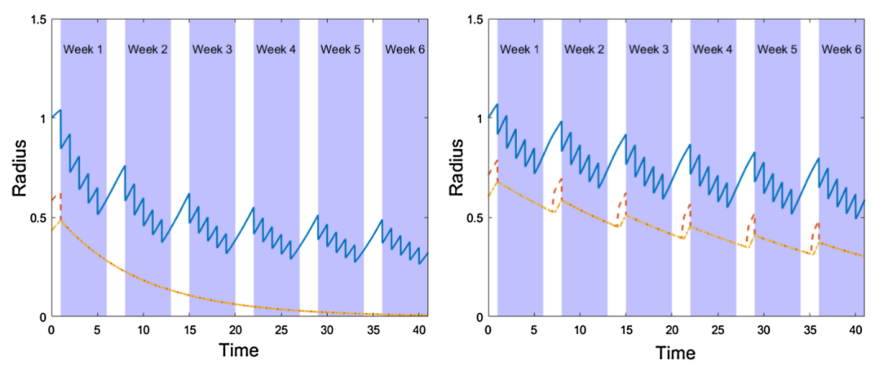

Fig. 5. Numerical solutions of the Greenspan model, showing how the response to a standard, 6-week, fractionation protocol (2 Gy fractions delivered daily Monday-Friday, shown as shaded regions) changes when the rate of natural, or apoptotic, cell death $\left(\mu_{A}\right)$ declines; at the end of treatment, the tumour with the smaller rate of natural cell death (right hand side) is larger and retains a necrotic core changes whereas the tumour with the larger rate of natural cell death (left hand side). Key: the outer tumour radius, $R(t)$ blue solid line; the hypoxic radius, $R_{H}(t)$-red dashed line; the necrotic core radius, $R_{N}(t)$-yellow dot/dashed line. Reproduced from [44], Figure 4.
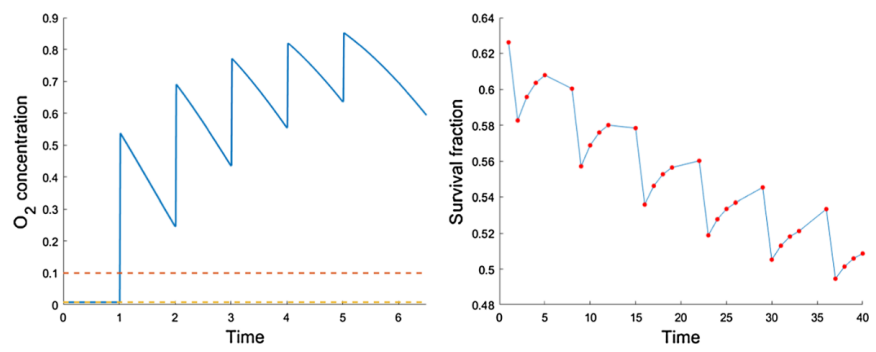

Fig. 6. Additional results associated with the simulation from the right hand side of Figure 5 Left hand side: this plot shows how, during the early stages of treatment, the oxygen concentration within the necrotic core fluctuates, and how, as the tumour shrinks in size, the average oxygen concentration rises above the necrotic and hypoxic thresholds, $c_{H}$ (dashed orange line) and $c_{N}$ (dashed yellow line). Right hand side: this plot shows how the volumeaveraged tumour survival fraction changes during the 6-week fractionation protocol. Key: red points correspond to the survival fraction when each radiotherapy dose is delivered, blue line added for ease of visualisation. Reproduced from [44], Figure 5.

structure evolves. Figure 6 shows how the oxygen concentration within the necrotic core fluctuates during the early stages of treatment. As successive doses of radiotherapy cause the tumour to shrink in size, oxygen levels at the centre of the necrotic core increase, eventually rising above the necrotic and hypoxic thresholds. As treatment progresses, reoxygenation of previously hypoxic and necrotic regions combined with an increase in the proportion of the tumour occupied by proliferating cells, leads to increased radiosensitivity and a corresponding reduction in the average survival fraction.

\section{B. The proliferation-invasion model}

While the Greenspan model incorporates heterogeneity due to spatial variations in oxygen levels, other types of heterogeneity are of interest when modelling tumour growth and radiotherapy. For example, when glioma cells, a common type of brain tumour, invade brain tissue, their motility and proliferation rates differ in grey and white matter. Swanson and colleagues [43], [45] proposed the following PDE model to describe how glioma cells move by random motion and 
proliferate in grey and white matter:

$$
\frac{\partial c}{\partial t}=\nabla \cdot(D(\mathbf{x}) \nabla c)+\rho c .
$$

In Equation 27), $c(\mathbf{x}, t)$ denotes the concentration of tumour cells at time $t$ and position $\mathbf{x}$, and $\rho$ is the net proliferation rate of the glioma cells. The diffusion coefficient $D(\mathbf{x})$ is piecewise constant, with $D(\mathbf{x})=D_{g}, D_{w}$ in grey and white matter, and $D_{w}>D_{g}$ to account for the greater motility of glioma cells in white matter.

Equation (27) can be extended to account for additional effects. For example, the net proliferation term $\rho c$ can be replaced with a logistic growth model, giving

$$
\frac{\partial c}{\partial t}=\nabla \cdot(D(\mathbf{x}) \nabla c)+\rho c\left(1-\frac{c}{K}\right),
$$

with carrying capacity $K$ and constant growth rate $\rho$ [46]. Rockne, Rockhill, Mrugala, et al. [47] use this formulation of the PI model to account for death from radiotherapy using the LQ model:

$$
\begin{aligned}
\frac{\partial c}{\partial t}=\nabla \cdot & (D \nabla c)+\rho c\left(1-\frac{c}{K}\right) \\
& -\left(1-e^{-\left(\alpha d+\beta d^{2}\right)}\right) c\left(1-\frac{c}{K}\right) \delta\left(t-t_{i}\right) .
\end{aligned}
$$

The PI model has also been extended to account for additional sources of environmental heterogeneity due to vascular remodelling, and, as with the Greenspan model, spatial variation in local oxygen levels. The resulting proliferationinvasion-hypoxia-necrosis-angiogenesis (PIHNA) model extends Equation (28) by including additional PDEs to describe the distributions of hypoxic glioma cells, necrotic matter, blood vessel density and angiogenic factors [46]. As with the modified Greenspan model, changes in the cell kill rate due to radiotherapy as oxygen levels vary can be incorporated via the modified LQ model defined by Equations (25) and (26) (see [48]).

\section{Model extensions}

As with the ODE models, the PDE models described above can be extended in multiple ways. Additional equations can be included to account for additional species (e.g., immune cells and blood vessels), and treatment combinations (e.g., radiotherapy, virotherapy, chemotherapy and/or immunotherapy [49]).

With increasing evidence that tumour cells are sensitive to mechanical stimuli, other authors have developed biomechanical models in which the tumour is viewed as a mixture of interacting phases. Mass and momentum balances are applied to each phase and appropriate constitutive laws are used to describe their mechanical properties. Multiphase models have been used to study vascular tumour growth [50] and the response of avascular tumours to radiotherapy and virotherapy [51], [52]. They have also been used to show how growth-induced compression of a compliant tissue matrix that surrounds a tumour spheroid can generate restraining forces which inhibit its growth, even when nutrients are freely available [53]. In future work, they could be used to study the efficacy of therapies designed to alter the mechanical properties of the tissue surrounding a tumour by, for example, neutralising the action of matrix-degrading proteases that facilitate continued tumour growth and invasion [9]. With their origins in physics, phase field models represent an exciting alternative to multiphase models [54], [55]. A key advantage of phase field models is their ability to accommodate topology changes caused by, for example, the disappearance of a tumour following successful radiotherapy, or the emergence of new blood vessels during angiogenesis.

\section{AGENT-BASED MODELS}

While PDE models can describe spatial heterogeneity, they are unable to account for some important aspects of tumour biology. In particular, they cannot resolve individual cells and therefore cannot explore situations for which cell level differences are important. Tumours are multiscale, and their growth dynamics and response to treatment are influenced by cross-talk between tissue-scale processes (e.g., angiogenesis, vascular remodelling and blood flow) and subcellular ones (e.g., cytokine production and the effect of local nutrient levels on cell cycle progression). In order to account for subcellular effects, discrete models which resolve individual cells have been developed. These models are often termed agent-based models (ABMs). They admit cell-scale spatial resolution and can accommodate detailed subcellular, cellcell and cell-microenvironmental interactions. Many ABMs combine continuum and discrete approaches to account for processes at different biological scales (and their interactions). Such hybrid models of tumour growth and radiotherapy typically treat cells as discrete entities and associate with them sub-cellular models which govern cell behaviour in response to microenvironmental cues and radiotherapy. The subcellular models may be stochastic or they may comprise deterministic ODEs. PDEs are commonly used to describe the distribution of nutrients such as oxygen and glucose (and chemotherapeutic drugs) at the tissue-scale, with the discrete cells acting as point sources or sinks. The spatial distributions of the diffusible species may, in turn, influence the behaviour of individual cells at the subcellular level. While hybrid models can be biologically detailed, their complexity comes with increasing computational cost and larger numbers of parameters, which can make them challenging to simulate, validate and fit to data.

The behaviour of the agents depends on the modelling framework. Several important choices must be made: whether to use an on-lattice framework (in which agents are confined to locations on a regular grid) or an off-lattice framework (in which agents move freely in space); whether to represent cells by the positions of their cell-centres or to explicitly account for their shape. Common choices of lattice-based models include cellular automata and cellular Potts models [56], while offlattice models include Voronoi methods, overlapping-spheres models, and vertex-based models [57].

While some ABMs are developed from scratch, it is increasingly common to use pre-existing software to construct ABMs. Exploiting existing software is particularly important when complex models are required, as errors in the underlying model implementation are less likely to be introduced and 


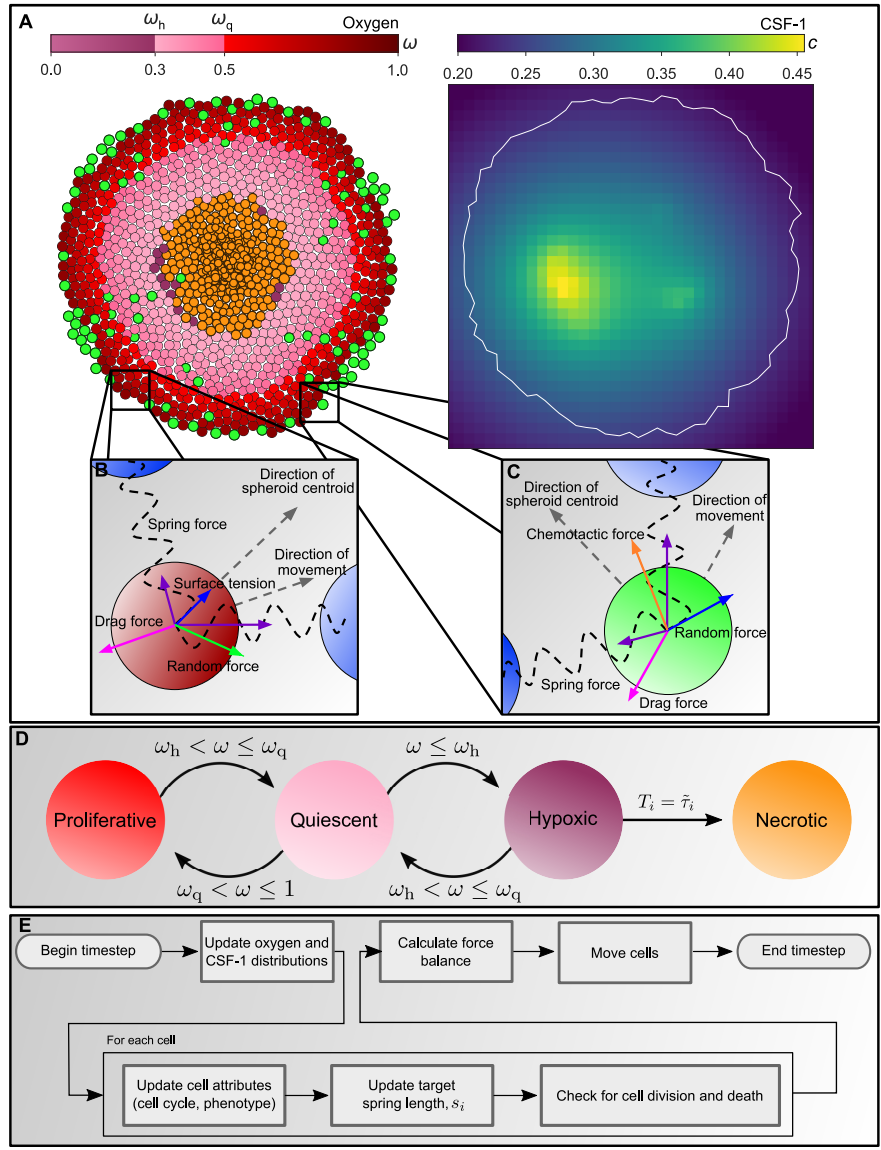

Fig. 7. Schematic diagram showing how tissue-level, cell-level and subcellular components may interact within an ABM. In this model, individual cells form a tumour spheroid (A) and consume oxygen as it diffuses from the spheroid boundary. The distributions of oxygen and the macrophage chemoattractant CSF-1 (produced by hypoxic tumour cells) are calculated at the tissue-level. Tumour cells (B) and macrophages (C) exert mechanical forces on each other Additional forces act on the agents, and may depend on the tissue-level chemical distributions: for example, the chemotactic force experienced by each macrophage depends on the local gradient of CSF-1. The net force on a cell determines its movement at each timestep. Panel (D) shows how local oxygen oxygen levels influence tumour cell phenotype at at the subcellular scale. The flow chart in panel (E) summarises how the ABM is updated on each timestep. Reproduced from [64], Figure S1.

more likely to be identified in large, open source software collaborations. Available software packages include: Chaste (Cancer, Heart and Soft Tissue Environment) [58], which implements a range of different ABMs; Physicell [59], which combines an overlapping spheres model for individual cells with BioFVM [60] to accommodate interactions between cells and multiple diffusible species such as oxygen; and HAL (Hybrid Automata Library) [61], a JAVA based library with modular hybrid modelling capabilities and efficient visualization tools. Other tools include Morpheus [62], and Biocellion [63].

In Figure 7 we present a schematic of an off-lattice ABM to show how such models are composed and how tissue, cell and subcellular processes may interact. The model describes macrophage infiltration into a tumour spheroid in response to spatial gradients of colony stimulating factor-1 (CSF-1), a chemoattractant produced by hypoxic tumour cells. At the tissue-level, PDEs describe the diffusive transport, production and consumption of oxygen and CSF-1, with tumour cells acting as point sinks of oxygen and point sources of CSF-1 if their local oxygen levels are low. The distributions of oxygen and CSF-1 modulate the cell and subcellular behaviours of the individual cells. For example, macrophages experience a 'chemotactic force' in the direction of increasing CSF-1 gradients. Additionally, the local oxygen concentration influences the tumour cells' phenotypes and, thereby, its progress through the cell cycle. Subcellular processes also influence cell-level processes, with new cells being produced at the end of the cell cycle, and interaction forces between neighbouring cells depending on their phenotypes.

In the remainder of this section, we present examples of both on- and off-lattice ABMs, and show how their increased spatial resolution allows more detailed descriptions of radiotherapy than is feasible with ODE and PDE models.

\section{A. On-lattice models}

On-lattice models restrict the location of agents to a discrete set of lattice points. These points are typically a square lattice, although triangular or hexagonal meshes are sometimes used. On-lattice ABMs have several advantages: they are relatively simple to implement and, therefore, computationally efficient to simulate. They leverage the power of an ABM to incorporate cell-scale spatial heterogeneity and subcellular phenomena without requiring detailed consideration of the biomechanical forces that act on individual cells.

Cellular automaton (CA) models are arguably the most common and simplest type of on-lattice models. They typically assume that each cell occupies a single lattice site. The occupancy of the lattice sites is updated at discrete timesteps, which may range from seconds to hours, depending on the biological processes of interest. For example, on a given timestep a tumour cell may attempt to proliferate with probability $p_{1}$ by placing a daughter cell into an adjacent empty lattice site, it may attempt to move to an adjacent empty site with probability $p_{2}$, and it may do nothing with probability $1-p_{1}-p_{2}$. The probabilities $p_{1}$ and $p_{2}$ may be fixed, or may depend on the subcellular status of an individual cell (e.g., proliferation may occur only if a cell has completed its cell cycle) and its local microenvironment (e.g., a cell's movement may be biased up spatial gradients of a chemoattractant). While many on-lattice models can be described as cellular automata, we note that cellular Potts models, in which each biological cell occupies multiple neighbouring lattice sites, represent an alternative onlattice framework that has also been used to simulate the effects of radiation therapy on tumours (see, e.g., [65]).

In the context of radiotherapy, on-lattice models that include subcellular cell-cycle models can include more detailed descriptions of cell kill in response to treatment. For example, Brüningk and colleagues use a $\mathrm{CA}$ framework to simulate therapies that combine radiation with hyperthermia [66]. The virtual cells in their model have an internal cell cycle consisting of G1, S, G2 and M-phases, and progress through the phases is based on an internal timer. Cells enter a quiescent G0 phase, during which their cell cycle is paused, if there 
are no free spaces in their neigbourhood, and resume cycling when free spaces become available. The LQ model used in continuum models to determine the fraction of cells that survive exposure to radiotherapy is replaced by a stochastic model which defines the probability that an individual cell survives exposure to a dose $d$ of radiotherapy. This model accounts for both local oxygen levels $c$ and cell cycle phase, $\gamma$ [66], and takes the form:

$$
S F(c)=\exp \left(-\gamma \cdot\left(\alpha \cdot \frac{d}{\mathrm{OER}(c)}+\beta \cdot \frac{d^{2}}{\mathrm{OER}^{2}(c)}\right)\right)
$$

where

$$
\operatorname{OER}(c)= \begin{cases}1, & \text { if } c>11 \mathrm{mmHg} \\ 3-\frac{2 \cdot c}{11} & \text { if } c \leq 11 \mathrm{mmHg} .\end{cases}
$$

An advantage of this model compared to the LQ model is that, following radiation damage, cell death is not necessarily instantaneous. Rather, when a dose of radiotherapy is given each agent samples a random number $N$ that lies between 0 and 1. If $N>S_{R T}$, the cell is labelled for death but only dies when the cell attempts to divide, at the end of the cell cycle. If, during this time, the cell becomes quiescent due to lack of oxygen, it remains alive until its cell cycle reaches the M-phase and then cell death is induced.

\section{B. Off-lattice models}

While cellular automata models provide a relatively computationally efficient means of introducing subcellular effects and stochasticity into models of cancer, they cannot describe complex spatial interactions between neighbouring cells or accurately account for the forces between them. These forces arise through mechanisms such as intercellular adhesion or volume exclusion. On-lattice models are generally unsuitable for exploring these types of interactions, and so off-lattice models in which agents are free to move around a domain without restriction to a lattice are used when these forces may be important. For instance, in the example shown in Figure 7. an off-lattice model was used since the model was required to incorporate the effects of tumour cells obstructing macrophage chemotaxis and of advective movement driven by spatially restricted proliferation and death of tumour cells. Off-lattice models are generally more computationally intensive than onlattice ones, but are able to capture a more realistic range of cell-scale interactions between individual cells.

For example, Kempf, Bleicher, and Meyer-Hermann [67] use a 3-D off-lattice model of tumour spheroid growth to understand how radiation may drive cell cycle synchronisation and how this could be exploited by subsequent radiation treatments to increase cell kill. They use a Voronoi-Delaunay approach in which the shapes of individual cells can be defined from the positions of their centres. A subcellular model governs progress through the cell cycle and includes two checkpoints at which the cell cycle can be paused. During the G2/M-phase, cells which have been damaged by radiation die, either immediately or via apoptosis at the G2 checkpoint. The second checkpoint is during the G1 phase, during which cells become quiescent if their internal pressure is sufficiently
A
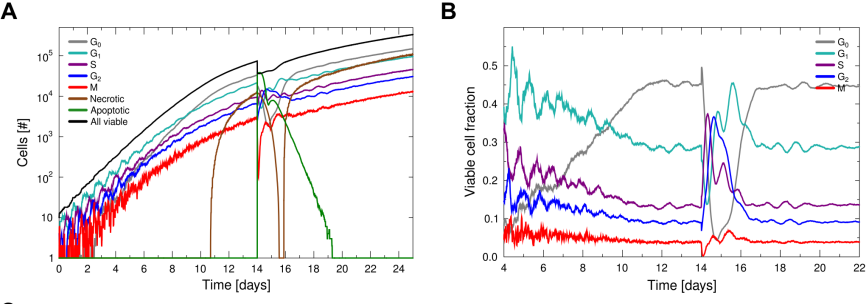

C

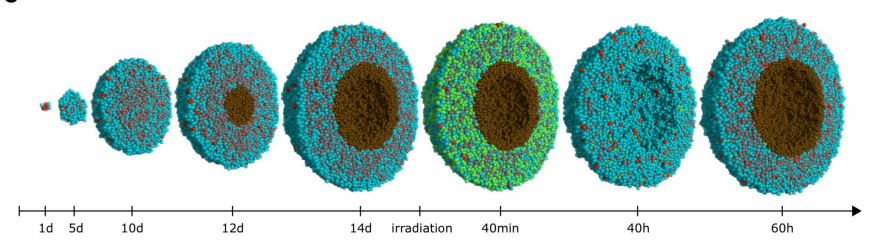

Fig. 8. Response of a spheroid to 4 Gy of radiation. Panels A and B show the effect of radiation on redistribution of cell cycles, one of the $5 \mathrm{R}$ 's of radiotherapy. Panel $\mathrm{C}$ shows a cut through the spheroid. A small number of cells rapidly grow into a solid tumour spheroid with quiescent cells in high density regions. Once the spheroid is large enough, nutrient deprivation causes cells in the centre to die and form a necrotic core. After irradiation many cells become apoptotic, leading to a gradual reduction in spheroid size and sufficient re-oxygenation to reactive quiescent cells. Finally, the cell density increases until the necrotic core re-establishes itself. Key: cyan $=$ G1, S or G2-phase, red = mitotic cells, grey = quiescent cells, brown = necrotic cells, green $=$ apoptotic cells. Figure reproduced from [68], Figure 2 .

high. This mechanism mimics contact inhibition of growth when cells experience high intercellular forces from their neighbours. As with the other models above, this model also accounts for cell death and quiescence due to insufficent levels of tissue-level concentrations of oxygen and glucose, their distributions being described via PDEs. A typical model simulation, showing how a tumour responds to irradiation, is presented in Figure 8. In common with the ABM models described above, Kempf, Bleicher, and Meyer-Hermann [67] use a variant of the linear-quadratic model to determine the cell survival fraction: they assume that the survival fraction depends on the cell cycle phase so that:

$$
S F_{p}=\exp \left(-\left(\alpha_{p} d+\beta_{p} d^{2}\right)\right),
$$

where $p$ indicates the cell cycle phase $(\mathrm{G} 1, \mathrm{~S}, \mathrm{G} 2$ or $\mathrm{M})$, and $\alpha_{p}$ and $\beta_{p}$ are cell cycle phase-specific constants.

\section{Model extensions}

The on- and off-lattice models described above show how ABMs can be used to add subcellular complexity to models of cancer growth and treatment. Due to their multiscale nature, these models can be extended to account for additional subcellular and tissue-scale behaviours as embodied in the Hallmarks of Cancer (see Figure 11). In this section we outline some extensions to ABMs that highlight this ability.

One of the Hallmarks of Cancer is "genome instability and mutation". Mutational status can be represented as a subcellular property of an ABM. For example, coupling subcellular process with ABMs is necessary to account for the emergence of resistance in cell populations. Resistance to therapy is gained via random mutations which confer an advantage to the resulting cell population. Since these mutations are stochastic, 


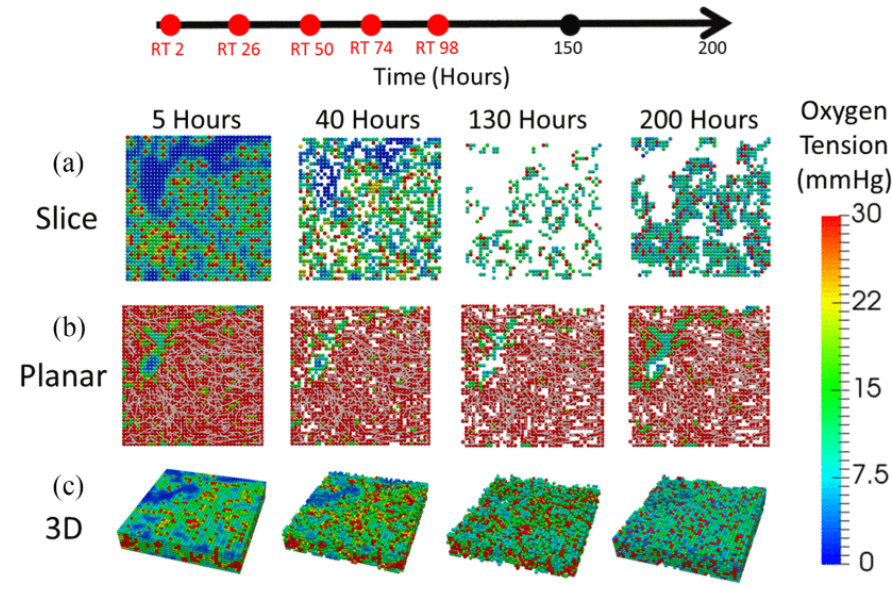

Fig. 9. Influence of geometry on cell growth and response to RT. Simulations at times $\mathrm{t}=5,40,130$ and $200 \mathrm{~h}$, comparing the response of tumor cells embedded in (a) "Slice," (b) "Planar," and (c) the "3-D" representation of one of the biological networks to five rounds of daily RT. Administration times are also indicated. Cellular oxygen tension in $\mathrm{mmHg}$ is contoured. Parameter values: as per Table I in [69], except for the oxygen consumption rate which is increased by a factor of 15 (from $\mathrm{k}=13 \mathrm{~min}-1$ to $\mathrm{k}=195 \mathrm{~min}-1$ ). Figure reproduced from [69], Figure 4.

the proportion of cells in a tumour which are resistant to treatment may initially be too small to accurately describe using continuum approaches. Similarly, if a mutation which confers radioresistance also reduces a cell's proliferation rate, then the mutant cell population may die out before treatment is applied. In this way, the stochasticity of an ABM may help explain why radiotherapy successfully eliminates some patients' tumours while, in other patients, small populations of radioresistant cells drive relapse following treatment.

A second hallmark of cancer, "inducing angiogenesis", suggests a way in which the ABMs described here could be extended at the tissue scale to show more detail by including vasculature. For example, Grogan and coworkers consider the impact of vascular geometry on cell growth and response to radiotherapy [69]. Figure 9 shows three representations of a vascular network. The different geometries are used to simulate the oxygen distribution within the tissue and the impact of radiotherapy on the constituent tumour cells. ABMs can also be coupled to detailed models of tumour blood flow, angiogenesis and vascular remodelling to understand how their impact on tumour heterogeneity and the emergence of hypoxic regions [70]. Such detailed ABMs also permit investigation of treatments in which radiotherapy is combined with hypoxiaactivated prodrugs [71].

\section{INTEGRATING MATHEMATICAL MODELS WITH DATA}

In sections III, IV and V, we introduced ODE, PDE and ABM models of solid tumour growth and focussed on the qualitative insight that they can generate. In order to realise their full potential, the models should be validated against appropriate data (see Figure 3). Until recently, this has proven a significant challenge to the integration of mathematical models with clinical decision making and/or experimental research. As we explain below, there are encouraging signs that the landscape is changing.
Although advances in medical imaging enable collection of more detailed data, most patient data is relatively low resolution. For example, routine CT scans provide approximate measurements of tumour volume, with high levels of uncertainty. Such data can be used to parameterise simple ODE models, but is inadequate for accurate parameterisation of spatial models. While higher resolution imaging techniques will aid parameterisation of more complex models, parameter estimation remains a major bottleneck to the wider integration of mathematical models into clinical practice. As such, more complex models are often calibrated against experimental data to estimate parameter values which subsequently inform clinical models [72]. Automated image analysis tools can also be integrated into the mathematical modelling process to enable more accurate data fitting and model selection [73].

Integrating models with clinical data can provide new insight in situations where treatment yields unexpected behaviours. For example, the patient data presented in Figure 10 does not conform to the 'saw-tooth' dynamics that singlecompartment ODE models produce (see Figure 4). In part, this difference is due to the time resolution of the clinical data. The data points in Figure 10 are separated by at least 1 week, whereas the saw-tooth dynamics occur on shorter timescales when radiotherapy is delivered daily. However, differences in temporal resolution cannot fully explain why the clinical data does not always conform to "saw-tooth" dynamics. For instance, in some patients, the tumour volume initially increases during treatment and only starts to decrease mid-way through treatment. Such dynamic behaviour cannot be captured by the saw-tooth dynamics depicted in Figures 4,5 and 6 . By fitting the ODE model defined by Equations 13 and 14 to this patient data, Lewin, Maini, Moros, et al. [41] showed that such behaviour may arise if dead cells are not removed instantaneously following radiotherapy. Figure 10 also shows the value of using Bayesian methods to obtain distributions of parameter estimates rather than specific, pointwise values, as these posterior distributions can be used to identify $95 \%$ credible intervals around the model predictions.

Although parameter estimation remains challenging, fitting patient-specific parameters to mathematical models provides an opportunity to personalise treatment [74], [75]. Enderling and colleagues are currently running a clinical trial (NCT03656133) in which the logistic growth model is fit to pre-treatment data to estimate patient-specific parameter values which are used to assign patients to different radiotherapy protocols (e.g., hypofractionation and hyperfractionation, when the same total dose of radiation is given in a large number of small doses or a small number of large doses) [32], [71]. Mathematical models can also be used to identify optimal treatment protocols which may differ from the standard of care. After fitting patient-specific parameters to a mathematical model, optimal control theory can be used to propose optimal treatments which, for example, maximise expected tumour volume reduction while minimising the total radiation dose applied to the patient [76].

Mathematical models can also be used to conduct 'virtual clinical trials' in which virtual patient cohorts, characterised by distributions of model parameter values, are exposed to 


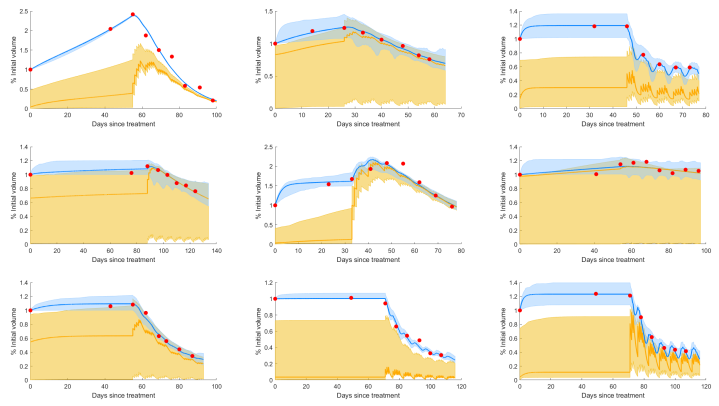

Fig. 10. Results obtained by fitting the two compartment model defined by Equations [13) and (14) to clinical patient data using the Approximate Bayesian Computation Sequential Monte Carlo (ABC SMC) algorithm. The blue and yellow curves show the total tumour and necrotic volume for simulations using the best-fitting parameter sets. At each time point, the $95 \%$ credible intervals for the total tumour and necrotic volume (blue and yellow regions, respectively) are obtained by sampling 120 parameter combinations from the posterior distributions. Clinical data points are marked by red filled circles. Figure reproduced from [41], Figure 3.

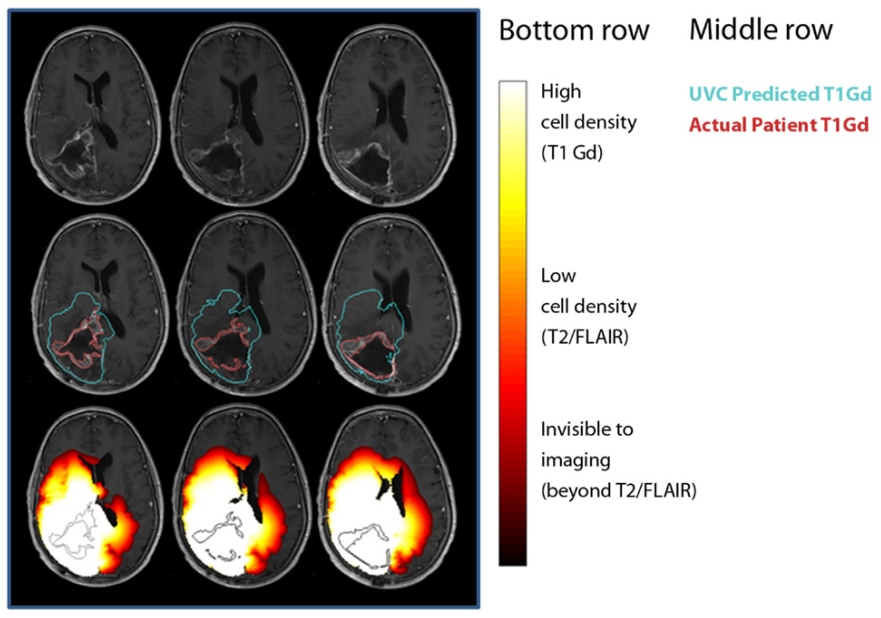

Fig. 11. Comparisons between untreated virtual controls and post-treatment MRI scans. First row: post-treatment MRI. Second row: contours showing measured tumor on T1-Gd-enhanced scan (red) and UVC prediction of T1Gd area (aqua). Third row: UVC tumor cell densities overlaid (white, high cell density; red, low cell density) on scan with T1-Gd measured tumor outline (black). Figure reproduced from [77], Figure 7.

different treatments parameters, and the expected range of treatment outcomes predicted, during trial design. Virtual trials can also be used to establish what data should be collected to reliably quantify patient responses to the different treatments. In this way, the targeted use of mathematical modelling has the potential to enable clinicians to identify and correct potential weaknesses in trial design.

As use of medical resonance imaging (MRI) and positon emission tomography increases, access to spatially-resolved data which can detect tumour boundaries and show how they change over time is becoming available to clinicians and mathematical modellers. As this data has limited spatial resolution and is unable to resolve individual cells, PDE models represent a natural framework for interpreting such images. For example, in Figure 11 the proliferation-invasion model was used to simulate glioma growth and model outputs compared to MRI images [77]. The mathematical model is termed an Untreated Virtual Control (UVC). The UVC uses pre-treatment scans to simulate how tumour development may have continued in the absence of treatment, allowing clinicians to assess how well treatment is working based on deviations between posttreatment measurement and the UVC. This example highlights a further benefit of using mathematical modelling in the clinic: since medical images have limited spatial resolution, they can only detect tumour regions where the density exceeds a threshold value. By contrast, mathematical models can 'see' below such threshold values, and more accurately predict the true extent of tumour invasion and spread.

Outside the clinic, techniques such as imaging mass cytometry and multiplex immunohistochemistry can generate spatial images that resolve the expression of specific proteins at cellular and subcellular resolution. By integrating such images with single-cell RNA sequencing data, it is now possible to collect exquisitely detailed experimental data at subcellular, cellular and tissue scales. Such data is ideally suited for parameterising and validating multiscale models which can be used to help develop new therapies and drive fundamental research into our understanding of tumour growth and development.

\section{CONCLUSIONS}

In this paper, we have reviewed a range of mathematical models that describe tumour growth and response to radiotherapy, and illustrated the mechanistic insight that they can generate. In the same way that Hanahan and Weinberg's Hallmarks of Cancer provide a guiding principle for characterising different cancers, we now propose six Hallmarks of Mathematical Oncology which can be used to characterise mathematical models: discrete or continuous variables, deterministic or stochastic, single- or multiscale, spatially-averaged or spatially-resolved, homogeneous or heterogeneous, and single or hybrid (see Figure 2).

Confronted by the array of mathematical approaches being used to study tumour growth, it can be difficult to know what approach and level of detail to use. The situation can be compounded when different models are equally compatible with the experimental data. In practice, such decisions depend on the questions under investigation and the available data. In general, cell-based models can be more easily extended to account for additional data on subcellular signalling pathways and/or the cell cycle, whereas coarser-grained PDE and spatially-averaged ODE models contain fewer parameters and, as a result, should yield more robust fits to the data. While complex models, with multiple parameters, may overfit experimental data, they can act as surrogates for experimental data with the advantage that there is a known "ground truth" against which to test the predictive accuracy of simpler models. Care must therefore be taken to balance model complexity against the availability of experimental data. Additionally, if model simulations from different approaches yield qualitatively different behaviour (such as their predicted response of a multicellular spheroid to exposure to a particular drug), then these can be used to design experiments that discriminate between the alternative models. 
In practice, close collaboration between theoreticians and biomedical researchers is crucial throughout this process, because the models are only ever as good as the assumptions used to construct them and the data with which they are validated. Indeed, in many respects the form of the initial model is less important than starting the dialogue between experimentalists and modellers because any model is almost certain to be wrong!

In conclusion, mathematical models represent a natural framework for not only testing biological hypotheses and generating new ones but also optimizing experimental protocols. As we survey the large body of research devoted to modelling tumour growth, it is clear that considerable progress has been made. Many mathematical and computational tools (or models) have been developed: what is now needed is to translate these prototypes into validated models that are specialized for particular tumours and treatments, and that have the power to generate qualitative and quantitative predictions. The increasing number of modelling papers appearing in the literature indicates that this transition is starting to happen.

\section{ACKNOWLEDGMENT}

This work was supported by Cancer Research UK (CRUK) grant no. C5255/A18085, through the CRUK Oxford Centre.

\section{REFERENCES}

[1] H. Sung, J. Ferlay, R. Siegel, et al., "Global cancer statistics 2020: GLOBOCAN estimates of incidence and mortality worldwide for 36 cancers in 185 countries.," CA: A Cancer Journal for Clinicians, vol. 71, pp. 209249, 3 2021. DOI: https://doi.org/10.3322/caac.21660.

[2] D. Hanahan and R. A. Weinberg, "The hallmarks of cancer," Cell, vol. 100, no. 1, pp. 57-70, 2000. [Online]. Available: http://www . ncbi .nlm . nih.gov/ pubmed/ 10647931

[3] — - "Hallmarks of cancer: the next generation," Cell, vol. 144, no. 5, pp. 646-674, 2011. DOI: 10.1016/j.cell. 2011.02.013. [Online]. Available: http://www.ncbi.nlm. nih.gov/pubmed/21376230.

[4] H. Enderling, J. C. L. Alfonso, E. Moros, J. J. Caudell, and L. B. Harrison, "Integrating Mathematical Modeling into the Roadmap for Personalized Adaptive Radiation Therapy," Trends in Cancer, vol. 5, pp. 467-474, 8 2019. DOI: 10.1016/j.trecan.2019.06.006.

[5] L. Preziosi, Cancer modelling and simulation. Chapman \& Hall / CRC Mathematical Biology Series Book 3, 2003.

[6] D. Wodarz and N. L. Komarova, Computational biology of cancer, Lecture Notes and Mathematical Modelling. World Scientific, 2005.

[7] J. Folkman and M. Hochberg, "Self-regulation of growth in three dimensions," J Experimental Medicine, vol. 138, pp. 745-753, 4 1973. DOI: 10.1084/jem.138. 4.745

[8] M. De Palma, D. Biziato, and T. Petrova, "Microenvironmental regulation of tumour angiogenesis," Nat Rev Cancer, vol. 17, pp. 457-474, 2017. DOI: $10.1038 /$ nrc. 2017.51
[9] W. Stetler-Stevenson, "The tumor microenvironment: regulation by MMP-independent effects of tissue inhibitor of metalloproteinases-2," Cancer Metastasis Rev., vol. 27, pp. 57-66, 1 2008. DOI: 10.1007/s10555007-9105-8.

[10] F. Perri, S. Pisconti, and G. D. V. Scarpati, "P53 mutations and cancer: a tight linkage," Annals of Translational Medicine, vol. 4, 24 2016. DOI: 10.21037/atm. 2016.12.40.

[11] I. Turesson, J. Carlsson, A. Brahme, et al., "Biological response to radiation therapy," Acta Oncologica, vol. 42, pp. 92-106, 2 2003. DOI: 10.1080/02841860310004959

[12] R. G. Dale, "Dose-rate effects in targeted radiotherapy.," Physics in medicine and biology, vol. 41, no. 10, pp. 1871-1884, 1996, ISSN: 0031-9155. DOI: 10.1088/ 0031-9155/41/10/001

[13] M. G. Haddock, "Intraoperative radiation therapy for colon and rectal cancers: A clinical review," Radiation Oncology, vol. 12, pp. 1-8, 1 2017. DOI: 10.1186/ s13014-016-0752-1

[14] G. G. Steel, "The search for therapeutic gain in the combination of radiotherapy and chemotherapy," Radiotherapy and Oncology, vol. 11, pp. 31-53, 1 1988. DOI: 10.1016/0167-8140(88)90044-8

[15] S. Brüningk, I. Rivens, C. Box, U. Oelfke, and ter Haar G., "3D tumour spheroids for the prediction of the effects of radiation and hyperthermia treatments," Scientific Reports, vol. 10, 1653 2020. DOI: 10.1038/ s41598-020-58569-4.

[16] M. Muthana, S. Rodrigues, Y.-Y. Chem, et al., "Macrophage delivery of an oncolytic virus abolishes tumor regrowth and metastasis after chemotherapy or irradiation," Cancer Research, vol. 73, pp. 490-495, 2 2013. DOI: 10.1158/0008-5472.CAN-12-3056.

[17] E. Van Limbergen, D. De Ruysscher, V. Olivo Pimentel, et al., "Combining radiotherapy with immunotherapy: the past, the present and the future," British Journal of Radiology, vol. 90, p. 20170 157, 1076 2017. DOI: 10.1259/bjr.20170157.

[18] P. Hoskin, M. Saunders, and S. Dische, "Hypoxic radiosensitizers in radical radiotherapy for patients with bladder carcinoma," Cancer, vol. 86, pp. 1322-1328, 7 1999. DOI: https://doi .org/10.1002/(SICI) 10970142(19991001)86:7〈1322::AID-CNCR30〉3.0.CO;2E

[19] M. Skwarski, E. Bowler, J. D. Wilson, G. S. Higgins, and E. M. Hammond, "Targeting tumor hypoxia," in Molecular Targeted Radiosensitizers: Opportunities and Challenges, H. Willers and I. Eke, Eds. Cham: Springer International Publishing, 2020, pp. 265-299, ISBN: 9783-030-49701-9. DOI: 10.1007/978-3-030-49701-9_11.

[20] D. Brenner, "The linear-quadratic model is an appropriate methodology for determining isoeffective doses at large doses per fraction," Seminars in radiation oncology, vol. 18, pp. 234-239, 4 2008. DOI: 10.1016/ j.semradonc.2008.04.004.

[21] D. J. Brenner, L. R. Hlatky, P. J. Hahnfeldt, Y. Huang, and R. K. Sachs, "The linear-quadratic model and most 
other common radiobiological models result in similar predictions of time-dose relationships.," Radiation research, vol. 150, no. 1, pp. 83-91, 1998, ISSN: 00337587. DOI: $10.2307 / 3579648$ arXiv: NIHMS150003.

[22] G. Steel, T. McMillan, and J. Peacock, "The 5Rs of Radiobiology," International Journal of Radiation Biology, vol. 56, pp. 1045-1048, 6 1989. Dor: 10.1080/ 09553008914552491 .

[23] E. Hall and A. Giaccia, Radiobiology for the Radiobiologist, 7th edition. Lippincott, Williams and Wilkins, 2011.

[24] M. Joiner and A. van der Kogel, Basic Clinical Radiobiology, 4th edition. Hodder Arnold, 2009.

[25] A. V. Ponce Bobadilla, P. Maini, and H. Byrne, "A stochastic model for tumour control probability that accounts for repair from sublethal damage," Math Med Biol, vol. 35, pp. 181-202, 2 2018. DOI: 10.1093/ imammb/dqw024.

[26] R. G. Dale, J. F. Fowler, and B. Jones, "A new incomplete-repair model based on a 'reciprocal-time' pattern of sublethal damage repair," Acta Oncologica, vol. 38, pp. 919-929, 7 1999. DOI: 10.1080/ 028418699432608

[27] A. Bertuzzi, A. Fasano, A. Gandolfi, and C. Sinisgalli, "Reoxygenation and split-dose response to radiation in a tumour model with Krogh-type vascular geometry," Bulletin of Mathematical Biology, vol. 70, no. 4, pp. 992-1012, 2008, ISSN: 00928240. DOI: 10.1007/ s11538-007-9287-9

[28] H. Enderling and M. Chaplain, "Mathematical modeling of tumor growth and treatment," Current Pharmaceutical Design, vol. 20, pp. 4934-40, 30 2014. DOI: 10. 2174/1381612819666131125150434

[29] J. Liu, D. Hormuth II, T. Davis, et al., "A time-resolved experimental - mathematical model for predicting the response of glioma cells to single-dose radiation therapy," Integrative Biology, vol. 13, pp. 167-183, 72021. DOI: 10.1093/intbio/zyab010

[30] P. Castorina, T. S. Deisboeck, P. Gabriele, and C. Guiot, "Growth laws in cancer: implications for radiotherapy," Radiation Research, vol. 168, pp. 349-356, 32007. DOI: $10.1667 /$ RR0787.1.

[31] M. U. Zahid, N. Mohsin, A. S. R. Mohamed, et al., "Forecasting Individual Patient Response to Radiation Therapy in Head and Neck Cancer With a Dynamic Carrying Capacity Model," International Journal of Radiation Oncology Biology Physics, vol. 111, pp. 693704, 3 2021. DOI: 10.1016/j.ijrobp.2021.05.132

[32] S. Prokopiou, E. G. Moros, J. Poleszczuk, et al., “A proliferation saturation index to predict radiation response and personalize radiotherapy fractionation," Radiation Oncology, vol. 10, no. 1, p. 159, 2015, ISSN: 1748717X. DOI: 10.1186/s13014-015-0465-x. [Online]. Available: http://www.ro-journal.com/content/10/1/159

[33] M. Paczkowski, W. W. Kretzschmar, B. Markelc, et al., "Reciprocal interactions between tumour cell populations enhance growth and reduce radiation sensitivity in prostate cancer," Commun Biol, vol. 4, pp. 1-13, 1 2021. DOI: 10.1038/s42003-020-01529-5

[34] P. Hahnfeldt, D. Panigrahy, J. Folkman, and L. Hlatky, "Tumor Development under Angiogenic Signaling," Cancer Research, vol. 59, pp. 4770-4775, 191999.

[35] R. K. Sachs, L. R. Hlatky, and P. Hahnfeldt, "Simple ODE models of tumor growth and anti-angiogenic or radiation treatment," Mathematical and Computer Modelling, vol. 33, pp. 1297-1305, 12-13 2001. DOI: 10. 1016/S0895-7177(00)00316-2

[36] V. A. Kuznetsov, I. Makalkin, M. Taylor, and A. Perelson, "Nonlinear dynamics of immunogenic tumors: parameter estimation and global bifurcation analysis," Bull. Math. Biol., vol. 56, pp. 295-321, 2 1994. DOI: 10.1007/BF02460644.

[37] I. Butuc, C. Mirestean, and D. Iancu, "A nonlinear model in the dynamics of tumour-immune system combined with radiotherapy," U.P.B. Sci. Bull., Series A, vol. 81, pp. 311-322, 42019.

[38] A. Ghaffari, B. Bahmaie, and M. Nazari, "A mixed radiotherapy and chemotherapy model for treatment of cancer with metastasis," Math. Meth. Appl. Sci., vol. 39, pp. 4603-4617, 2016. DOI: 10.1002/mma.3887.

[39] S. M. Al-Tuwairqi, N. O. Al-Johani, and E. A. Simbawa, "Modeling dynamics of cancer radiovirotherapy," Journal of Theoretical Biology, vol. 506, 1104052020. DOI: $10.1016 /$ j.jtbi.2020.110405.

[40] A. Chvetsov, S. Yartsev, J. Schwartz, and N. Mayr, "Assessment of interpatient heterogeneity in tumor radiosensitivity for nonsmall cell lung cancer using tumor-volume variation data," Medical Physics, vol. 41, p. 064 101, 6 2014. DOI: 10.1118/1.4875686.

[41] T. Lewin, P. Maini, E. Moros, J. Caudell, H. Enderling, and H. Byrne, "Using mathematical modelling to identify data requirements for increased prediction accuracy in radiotherapy," Scientific Reports (under review), 2021.

[42] H. P. Greenspan, "Models for the Growth of a Solid Tumor by Diffusion," Studies in Applied Mathematics, vol. 51, pp. 317-340, 4 1972. DOI: $10.1002 /$ sapm1972514317

[43] K. R. Swanson, E. C. Alvord Jr., and J. D. Murray, “A quantitative model for differential motility of gliomas in grey and white matter," Cell Prolif, vol. 33, no. 5, pp. 317-329, 2000. [Online]. Available: http://www. ncbi.nlm.nih.gov/pubmed/11063134.

[44] T. Lewin, P. K. Maini, E. G. Moros, H. Enderling, and H. M. Byrne, "The Evolution of Tumour Composition During Fractionated Radiotherapy: Implications for Outcome," Bulletin of Mathematical Biology, vol. 80, pp. 1207-1235, 5 2018. DOI: 10.1007/s11538-0180391-9.

[45] K. R. Swanson, E. C. Alvord Jr., and J. D. Murray, "Virtual brain tumours (gliomas) enhance the reality of medical imaging and highlight inadequacies of current therapy," Br J Cancer, vol. 86, no. 1, pp. 14-18, 2002. DOI: 10.1038/sj.bjc.6600021. [Online]. Available: http: //www.ncbi.nlm.nih.gov/pubmed/11857005 
[46] K. R. Swanson, R. C. Rockne, J. Claridge, M. A. Chaplain, E. C. Alvord, and A. R. A. Anderson, "Quantifying the role of angiogenesis in malignant progression of gliomas: In Silico modeling integrates imaging and histology," Cancer Research, vol. 71, pp. 7366-7375, 24 2011. DoI: 10.1158/0008-5472.CAN-11-1399.

[47] R. Rockne, J. K. Rockhill, M. Mrugala, et al., "Predicting the efficacy of radiotherapy in individual glioblastoma patients in vivo: a mathematical modeling approach.," Physics in medicine and biology, vol. 55, no. 12 , pp. $3271-3285$, 2010, ISSN: $1361-6560$. DOI: $10.1088 / 0031-9155 / 55 / 12 / 001$

[48] A. Hawkins-Daarud, R. Rockne, D. Corwin, A. R. A. Anderson, P. Kinahan, and K. R. Swanson, "In silico analysis suggests differential response to bevacizumab and radiation combination therapy in newly diagnosed glioblastoma," Journal of the Royal Society Interface, vol. 12, p. 20150388,109 2015. DOI: 10.1098/rsif. 2015.0388

[49] K. Storey, S. Lawler, and T. Jackson, "Modeling Oncolytic Viral Therapy, Immune Checkpoint Inhibition, and the Complex Dynamics of Innate and Adaptive Immunity in Glioblastoma Treatment," Frontiers in Physiology, vol. 11, pp. 1-18, 151 2020. DOI: 10.3389/ fphys.2020.00151

[50] M. Hubbard and H. Byrne, "Multiphase modelling of vascular tumour growth in two spatial dimensions," Journal of Theoretical Biology, vol. 316, pp. 70-89, 2013. DOI: 10.1016/j.jtbi.2012.09.031.

[51] T. Lewin, H. Byrne, P. Maini, J. Caudell, E. Moros, and H. Enderling, "The importance of dead material within a tumour on the dynamics in response to radiotherapy," Physics in Medicine and Biology, vol. 65, p. 015007 , 1 2020. DOI: 10.1088/1361-6560/ab4c27.

[52] M. Boemo and H. Byrne, "Mathematical modelling of a hypoxia-regulated oncolytic virus delivered by tumourassociated Macrophages," Journal of Theoretical Biology, vol. 461, pp. 102-116, 2019. DOI: 10.1016/j.jtbi. 2018.10.044.

[53] C. Chen, H. Byrne, and J. King, "The influence of growth-induced stress from the surrounding medium on the development of multicell spheroids," Journal of Mathematical Biology, vol. 43, pp. 191-220, 2001. DOI: 10.1007/s002850100091.

[54] M. Fritz, E. Lima, V. Nikolić, J. Tinsley Oden, and B. Wohlmuth, "Local and nonlocal phase-field models of tumor growth and invasion due to ECM degradation," Mathematical Models and Methods in Applied Sciences, vol. 29, pp. 2433-2468, 13 2019. DOI: 10.1142/ S0218202519500519.

[55] G. Vilanova, I. Colominas, and H. Gomez, "A mathematical model of tumour angiogenesis: growth, regression and regrowth," J. R. Soc. Interface, vol. 14, p. 20160918,20160918 2017. DOI: 10.1098/rsif.2016. 0918

[56] F. Graner and J. A. Glazier, "Simulation of Biological Cell Sorting Using a Two-Dimensional Extended Potts
Model," Physical Review Letters, vol. 69, pp. 20132017, 13 1992. DOI: 10.1103/PhysRevLett.69.2013.

[57] J. M. Osborne, A. Fletcher, J. Pitt-Francis, P. Maini, and D. J. Gavaghan, "Comparing individual-based approaches to modelling the self-organization of multicellular tissues," PLoS Computational Biology, vol. 13, pp. 1-34, 2 2017. DOI: 10.1371/journal.pcbi.1005387.

[58] F. Cooper, R. Baker, M. Bernabeu, et al., "Chaste: Cancer, Heart and Soft Tissue Environment," Journal of Open Source Software, vol. 5, p. 1848, 47 2020. DOI: 10.21105/joss.01848.

[59] A. Ghaffarizadeh, R. Heiland, S. Friedman, and M. Shannon, "PhysiCell : an Open Source Physics-Based Cell Simulator for 3-D Multicellular Systems," PLoS Computational Biology, vol. 14, pp. 1-34, 2 2018. DOI: 10.1371/journal.pcbi.1005991

[60] A. Ghaffarizadeh, S. Friedman, and P. MacKlin, "BioFVM: An efficient, parallelized diffusive transport solver for 3-D biological simulations," Bioinformatics, vol. 32, pp. 1256-1258, 8 2016. DOI: 10.1093/ bioinformatics/btv730.

[61] R. Bravo, E. Baratchart, J. West, et al., "Hybrid Automata Library: A flexible platform for hybrid modeling with real-time visualization," PLoS Computational Biology, vol. 16, e1007635, 3 2020. DOI: 10.1371/journal. pcbi. 1007635

[62] J. Starruß, W. De Back, L. Brusch, and A. Deutsch, "Morpheus: A user-friendly modeling environment for multiscale and multicellular systems biology," Bioinformatics, vol. 30, pp. 1331-1332, 9 2014. DOI: 10.1093/ bioinformatics/btt772

[63] S. Kang, S. Kahan, J. McDermott, N. Flann, and I. Shmulevich, "Biocellion: Accelerating computer simulation of multicellular biological system models," Bioinformatics, vol. 30, pp. 3101-3108, 21 2014. DOI: 10. 1093/bioinformatics/btu498

[64] O. Vipond, J. Bull, P. Macklin, et al., "Multiparameter persistent homology landscapes identify immune cell spatial patterns in tumors," Proceedings of the National Academy of Sciences, vol. 118, e2102166118, 412021. DOI: $10.1073 /$ pnas.2102166118.

[65] X. Gao, J. T. McDonald, L. Hlatky, and H. Enderling, "Acute and Fractionated Irradiation Differentially Modulate Glioma Stem Cell Division Kinetics," Cancer Research, vol. 73, pp. 1481-1490, 5 2013. DOI: 10. 1158/0008-5472.CAN-12-3429

[66] S. C. Brüningk, P. Ziegenhein, I. Rivens, U. Oelfke, and G. ter Haar, "A cellular automaton model for spheroid response to radiation and hyperthermia treatments," Scientific Reports, vol. 9, pp. 1-12, 17674 2019. DOI: 10.1038/s41598-019-54117-X.

[67] H. Kempf, M. Bleicher, and M. Meyer-Hermann, "Spatio-temporal cell dynamics in tumour spheroid irradiation," European Physical Journal D, vol. 60, no. 1, pp. 177-193, 2010, ISSN: 14346060. DOI: 10.1140/epjd/ e2010-00178-4.

[68] H. Kempf, H. Hatzikirou, M. Bleicher, and M. MeyerHermann, "In Silico Analysis of Cell Cycle Synchroni- 
sation Effects in Radiotherapy of Tumour Spheroids," PLoS Computational Biology, vol. 9, no. 11, 2013, ISSN: 1553734X. DOI: 10.1371/journal.pcbi.1003295.

[69] J. Grogan, B. Markelc, A. J. Connor, et al., "Predicting the influence of microvascular structure on tumour response to radiotherapy," IEEE Transactions on Biomedical Engineering, vol. 64, pp. 504-511, 3 2017. DOI: 10.1109/TBME.2016.2606563.

[70] J. A. Grogan, A. J. Connor, B. Markelc, et al., "Microvessel Chaste: An Open Library for Spatial Modeling of Vascularized Tissues," Biophysical Journal, vol. 112, pp. 1767-1772, 9 2017. DOI: 10.1016/j.bpj.2017.03. 036.

[71] S. Hamis, M. Kohandel, L. J. Dubois, Y. A., P. Lambin, and G. G. Powathil, "Combining hypoxia-activated prodrugs and radiotherapy in silico: Impact of treatment scheduling and the intra-tumoural oxygen landscape," PLOS Computational Biology, vol. 16, e1008041, 8 2020. DOI: 10.1371/journal.pcbi.1008041.

[72] E. Lima, N. Ghousifam, A. Ozkan, et al., "Calibration of Multi-Parameter Models of Avascular Tumor Growth Using Time Resolved Microscopy Data,' Scientific Reports, vol. 8, p. 14 558, 1 2018. DOI: 10.1038/s41598018-32347-9

[73] J. Yang, J. Virostko, D. Hormuth II, et al., "An experimental-mathematical approach to predict tumor cell growth as a function of glucose availability in breast cancer cell lines," PLoS ONE, vol. 16, e0240765, 7 2021. DOI: 10.1371/journal.pone.0240765.

[74] J. West, M. Dinh, J. Brown, J. Zhang, A. Anderson, and R. Gatenby, "Multidrug cancer therapy in metastatic castrate-resistant prostate cancer: An evolution-based strategy," Clinical Cancer Research, vol. 25, pp. 44134421, 14 2019. DOI: 10.1158/1078-0432.CCR-19-0006.

[75] J. Cunningham, F. Thuijsman, R. Peeters, et al., "Optimal control to reach eco-evolutionary stability in metastatic castrate-resistant prostate cancer," PLoS One, vol. 15, e0243386, 12 2020. DOI: 10.1371/journal.pone. 0243386

[76] U. Ledzewicz, H. Maurer, and H. Schättler, "Optimal Combined Radio- and Anti-Angiogenic Cancer Therapy," Journal of Optimization Theory and Applications, vol. 180, pp. 321-340, 2019. DOI: $10.1007 /$ s10957018-1426-y

[77] A. Baldock, R. Rockne, A. Boone, et al., "From patient-specific mathematical neuro-oncology to precision medicine," Frontiers in Oncology, vol. 3, p. 62, 2013. DOI: 10.3389/fonc.2013.00062.

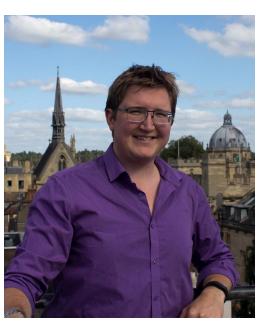

Joshua Adam Bull Joshua Bull received a DPhil through the Systems Approaches to Biomedical Science doctoral training centre at the University of Oxford, after a BA in Philosophy and Mathematics at the University of Southampton and an MSc in Mathematical Sciences at Durham University. He works as a postdoctoral researcher at the University of Oxford's Mathematical Institute, where his research focusses on developing novel mathematical and statistical approaches for image analysis and statistical description of multiplexed histological images. He also has strong interests in multiscale modelling and the integration of mathematical models with biomedical imaging data.

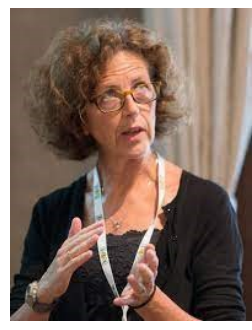

Helen Mary Byrne Helen Byrne has an MA in Mathematics from the University of Cambridge, and a Master of Science and DPhil from the University of Oxford. After postdoctoral research at the Universities of Oxford and Bath, she held lectureships at the UMIST in Manchester and the University of Nottingham, and was promoted to Professor in 2003. She was awarded an Advanced Research Fellowship from the United Kingdom's Engineering and Physical Sciences Research Council (2000-2006). In Nottingham, she established and then led the Centre for Mathematical Medicine and Biology before returning to Oxford in 2011 where she is now based in the Mathematical Institute. Helen's research is focussed on the development, analysis and simulations of continuum and multiscale models of biomedical systems, with particular emphasis on the growth and response to treatment of solid tumours. She received the Leah Edelstein-Keshet Award and was made a Fellow of the Society for Mathematical Biology for her contributions to mathematical biology. 